Supporting Information for:

\title{
An Efficient Route for the Synthesis of Glycosyl Phosphinic Acids
}

\author{
Charla A. Centrone and Todd L. Lowary* \\ Department of Chemistry, The Ohio State University \\ 100 West 18th Avenue, Columbus, Ohio 43210
}

\section{Table of Contents}

Experimental general methods and data for new compounds.

S2】S7

${ }^{1} \mathrm{H},{ }^{13} \mathrm{C}$, and ${ }^{31} \mathrm{P}-\mathrm{NMR}$ spectra for new compounds.

$\mathrm{S} 8 \square \mathrm{S} 45$ 
General. Solvents were distilled from the appropriate drying agents before use. Unless stated otherwise, all reactions were carried out at room temperature under a positive pressure of argon and were monitored by TLC on silica gel $60 \mathrm{~F}_{254}$. Spots were detected under UV light or by charring with $10 \% \mathrm{H}_{2} \mathrm{SO}_{4}$ in ethanol. Solvents were evaporated under reduced pressure and below $40{ }^{\circ} \mathrm{C}$ (bath). Organic solutions of crude products were dried over anhydrous $\mathrm{Na}_{2} \mathrm{SO}_{4}$. Unless otherwise indicated, column chromatography was performed on silica gel $60(40 \square 60 \square \mathrm{M})$. The ratio between silica gel and crude product ranged from 100 to 50:1 (w/w). Optical rotations were measured at $21 \pm 2{ }^{\circ} \mathrm{C}$. ${ }^{1} \mathrm{H}$ NMR spectra were recorded at 400 or $500 \mathrm{MHz}$, and chemical shifts are referenced to either TMS $\left(0.0, \mathrm{CDCl}_{3}, \mathrm{C}_{5} \mathrm{D}_{5} \mathrm{~N}\right) .{ }^{13} \mathrm{C}$ NMR spectra were recorded at 100 or $125 \mathrm{MHz}$ and chemical shifts are referenced to $\mathrm{CDCl}_{3}\left(77.00, \mathrm{CDCl}_{3}\right)$ or TMS $(0.0$, $\left.\mathrm{C}_{5} \mathrm{D}_{5} \mathrm{~N}\right) .{ }^{31} \mathrm{P}$ NMR spectra were recorded at 162 or $202 \mathrm{MHz}$ and chemical shifts are referenced to external phosphoric acid $\left(0.0, \mathrm{CDCl}_{3}, \mathrm{C}_{5} \mathrm{D}_{5} \mathrm{~N}\right)$. Electrospray mass spectra were recorded on samples suspended in mixtures of THF and $\mathrm{CH}_{3} \mathrm{OH}$ with added trifluoroacetic acid or $\mathrm{NaCl}$.

1-(Octyl)-2,5-anhydroglucityl phosphinic acid (21a). $R_{f} 0.45\left(1: 1, \mathrm{CH}_{2} \mathrm{Cl}_{2}\right.$ : $\left.\mathrm{CH}_{3} \mathrm{OH}\right) ;[\square]_{\mathrm{D}}+10.4\left(c 1.0, \mathrm{CH}_{3} \mathrm{OH}\right) ;{ }^{1} \mathrm{H}$ NMR $\left(500 \mathrm{MHz}, \mathrm{C}_{5} \mathrm{D}_{5} \mathrm{~N}\right) \mathrm{L}_{\mathrm{H}} 0.82(\mathrm{t}, 3 \mathrm{H}, J=7.1$ Hz), 1.40ワ1.11 (m, $10 \mathrm{H}), 2.09 \square 1.78$ (m, $4 \mathrm{H}), 2.72$ (br s, $2 \mathrm{H}), 4.29 \square 4.13$ (m, $2 \mathrm{H}), 4.47$ (br s, $1 \mathrm{H}), 4.76$ (br s, $1 \mathrm{H}), 4.84$ (br s, $1 \mathrm{H}), 5.10$ (br s, $1 \mathrm{H}), 8.29$ (br s, $4 \mathrm{H}) ;{ }^{13} \mathrm{C}$ NMR $\left(125 \mathrm{MHz}, \mathrm{C}_{5} \mathrm{D}_{5} \mathrm{~N}\right) \square_{\mathrm{c}} 14.3,22.9,29.5,29.6,31.2(\mathrm{~d}, J=88.0 \mathrm{~Hz}), 31.4(\mathrm{~d}, J=13.9 \mathrm{~Hz})$, $31.4(\mathrm{~d}, J=93.8 \mathrm{~Hz}), 32.1,63.4,77.9,79.8,79.9,87.5 ;{ }^{31} \mathrm{P} \mathrm{NMR}\left(202.4 \mathrm{MHz}, \mathrm{C}_{5} \mathrm{D}_{5} \mathrm{~N}\right) \square_{p}$ 49.6; HRMS calcd for $\mathrm{C}_{14} \mathrm{H}_{29} \mathrm{O}_{6} \mathrm{PNa}^{+}$: 347.1594. Found: 347.1607 . 
1-(Decyl)-2,5-anhydroglucityl phosphinic acid (21b). $R_{f} 0.38 \quad(2: 1$, $\left.\mathrm{CH}_{2} \mathrm{Cl}_{2}: \mathrm{CH}_{3} \mathrm{OH}\right) ;[\square]_{\mathrm{D}}+6.2\left(c 1.0, \mathrm{CH}_{3} \mathrm{OH}\right) ;{ }^{1} \mathrm{H} \mathrm{NMR}\left(500 \mathrm{MHz}, \mathrm{C}_{5} \mathrm{D}_{5} \mathrm{~N}\right) \square_{\mathrm{H}} 0.86(\mathrm{t}, 3 \mathrm{H}$, $J=7.1 \mathrm{~Hz}), 1.42 \square 1.18(\mathrm{~m}, 14 \mathrm{H}), 2.09 \square 1.79(\mathrm{~m}, 4 \mathrm{H}), 2.72$ (br. s, $2 \mathrm{H}), 4.16$ (dd, $1 \mathrm{H}, J=$ 11.1, $4.0 \mathrm{~Hz}$ ), 4.24 (dd, $1 \mathrm{H}, J=11.4,3.2 \mathrm{~Hz}$ ), 4.47 (br. s, $1 \mathrm{H}$ ), 4.75 (br. s, $1 \mathrm{H}$ ), 4.83 (br. s, $1 \mathrm{H}), 5.16 \square 5.08(\mathrm{~m}, 1 \mathrm{H}), 8.93$ (br. s, $2 \mathrm{H}) ;{ }^{13} \mathrm{C} \mathrm{NMR}\left(125 \mathrm{MHz}, \mathrm{C}_{5} \mathrm{D}_{5} \mathrm{~N}\right) \square_{\mathrm{c}} 14.4,23.0$, 29.6, 29.7, 29.7, 29.9, 30.1, 30.8, $31.2(\mathrm{~d}, J=92.4 \mathrm{~Hz}), 31.4(\mathrm{~d}, J=93.6 \mathrm{~Hz}), 31.5(\mathrm{~d}, J=$ 13.7 Hz), 32.2, 63.4, 78.0, 79.8, 80.0, 87.5; ${ }^{31} \mathrm{P}$ NMR (202.4 MHz, $\left.\mathrm{C}_{5} \mathrm{D}_{5} \mathrm{~N}\right) \square_{\mathrm{P}} 49.7$; HRMS calcd for $\mathrm{C}_{16} \mathrm{H}_{33} \mathrm{O}_{6} \mathrm{PNa}^{+}: 375.1907$. Found: 375.1889 .

1-(Dodecyl)-2,5-anhydroglucityl phosphinic acid (21c). $R_{f} 0.50$ (2:1, $\left.\mathrm{CH}_{2} \mathrm{Cl}_{2}: \mathrm{CH}_{3} \mathrm{OH}\right) ;[\square]_{\mathrm{D}}+7.2\left(c 1.1, \mathrm{CH}_{3} \mathrm{OH}\right) ;{ }^{1} \mathrm{H} \mathrm{NMR}\left(500 \mathrm{MHz}, \mathrm{C}_{5} \mathrm{D}_{5} \mathrm{~N}\right) \square_{\mathrm{H}} 0.87(\mathrm{t}, 3 \mathrm{H}$, $J=7.1 \mathrm{~Hz}$ ), $1.41 \square 1.15$ (m, $18 \mathrm{H}), 2.09 \square 1.76$ (m, $4 \mathrm{H}), 2.71$ (br. s, $2 \mathrm{H}), 4.28 \square 4.10$ (m, 2 H), 4.47 (br. s, $1 \mathrm{H}$ ), 4.75 (br. s, $1 \mathrm{H}$ ), 4.82 (br. s, $1 \mathrm{H}$ ), 5.10 (br. s, $1 \mathrm{H}$ ), 8.00 (br. s, $4 \mathrm{H}$ ); ${ }^{13} \mathrm{C}$ NMR $\left(125 \mathrm{MHz}, \mathrm{C}_{5} \mathrm{D}_{5} \mathrm{~N}\right) \square_{\mathrm{C}} 14.4,23.1,29.8,29.8,30.0,30.1,30.1,30.9,31.5(\mathrm{~d}, J=$ 89.0 Hz), $31.6(\mathrm{~d}, J=13.9 \mathrm{~Hz}), 32.3,63.5,78.0,79.9,80.0,87.6 ;{ }^{31} \mathrm{P}$ NMR $(202.4 \mathrm{MHz}$, $\left.\mathrm{C}_{5} \mathrm{D}_{5} \mathrm{~N}\right) \square_{p} 49.9$; HRMS calcd for $\mathrm{C}_{18} \mathrm{H}_{37} \mathrm{O}_{6} \mathrm{PNa}^{+}$: 403.2220. Found: 403.2218.

1-(Hexadecyl)-2,5-anhydroglucityl phosphinic acid (21d). $R_{f} 0.60(2: 1$, $\left.\mathrm{CH}_{2} \mathrm{Cl}_{2}: \mathrm{CH}_{3} \mathrm{OH}\right) ;{ }^{1} \mathrm{H}$ NMR $\left(500 \mathrm{MHz}, \mathrm{C}_{5} \mathrm{D}_{5} \mathrm{~N}\right) \square_{\mathrm{H}} 0.85(\mathrm{t}, 3 \mathrm{H}, J=7.1 \mathrm{~Hz}), 1.42 \square 1.19(\mathrm{~m}$, $26 \mathrm{H}), 2.05 \square 1.78(\mathrm{~m}, 4 \mathrm{H}), 2.79 \square 2.66(\mathrm{~m}, 2 \mathrm{H}), 4.16(\mathrm{dd}, 1 \mathrm{H}, J=11.2,4.5 \mathrm{~Hz}), 4.26(\mathrm{dd}$, $1 \mathrm{H}, J=11.4,4.0 \mathrm{~Hz}$ ), 4.49-4.46 (m, $1 \mathrm{H}), 4.76$ (br. s, $1 \mathrm{H}), 4.84$ (br. s, $1 \mathrm{H}), 5.13 \square 5.07$ (m, $1 \mathrm{H}), 7.56$ (br. s, $5 \mathrm{H}) ;{ }^{13} \mathrm{C}$ NMR $\left(125 \mathrm{MHz}, \mathrm{C}_{5} \mathrm{D}_{5} \mathrm{~N}\right) \square_{\mathrm{c}} 14.4,23.1,29.8,29.8,30.0$, $30.1,31.5(\mathrm{~d}, J=88.2 \mathrm{~Hz}), 31.5(\mathrm{~d}, J=14.8 \mathrm{~Hz}), 31.6(\mathrm{~d}, J=93.8 \mathrm{~Hz}), 32.3,63.5,78.1$, $79.9(\mathrm{~d}, J=4.8 \mathrm{~Hz}), 80.0,80.0,87.6 ;{ }^{31} \mathrm{P} \mathrm{NMR}\left(202.4 \mathrm{MHz}, \mathrm{C}_{5} \mathrm{D}_{5} \mathrm{~N}\right) \square_{\mathrm{P}} 49.4$; HRMS calcd for $\mathrm{C}_{22} \mathrm{H}_{45} \mathrm{O}_{6} \mathrm{PNa}^{+}$: 459.2846. Found: 459.2822 . 
1-(Eicosanyl)-2,5-anhydroglucityl phosphinic acid $(21 \mathrm{e}) . \quad R_{f} 0.62(2: 1$, $\left.\mathrm{CH}_{2} \mathrm{Cl}_{2}: \mathrm{CH}_{3} \mathrm{OH}\right) ;{ }^{1} \mathrm{H}$ NMR $\left(500 \mathrm{MHz}, \mathrm{C}_{5} \mathrm{D}_{5} \mathrm{~N}\right) \square_{\mathrm{H}} 0.86(\mathrm{t}, 3 \mathrm{H}, J=7.1 \mathrm{~Hz}), 1.45 \square 1.18(\mathrm{~m}$, $34 \mathrm{H}), 2.06 \square 1.79(\mathrm{~m}, 4 \mathrm{H}), 2.80 \square 2.69(\mathrm{~m}, 2 \mathrm{H}), 4.17(\mathrm{dd}, 1 \mathrm{H}, J=11.5,4.7 \mathrm{~Hz}), 4.27$ (dd, $1 \mathrm{H}, J=11.5,4.5 \mathrm{~Hz}), 4.51 \square 4.46(\mathrm{~m}, 1 \mathrm{H}), 4.79 \square 4.76(\mathrm{~m}, 1 \mathrm{H}), 4.87 \square 4.83(\mathrm{~m}, 1 \mathrm{H})$,

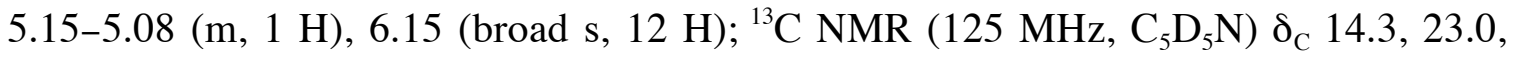
29.6, 29.7, 29.9, 29.9, 30.0, 30.0, $31.3(\mathrm{~d}, J=87.7 \mathrm{~Hz}), 31.4(\mathrm{~d}, J=14.9 \mathrm{~Hz}), 31.5(\mathrm{~d}, J=$ 93.2 Hz), 32.1, 63.3, 77.9, 79.8, $79.9(\mathrm{~d}, J=4.9 \mathrm{~Hz}), 87.4 ;{ }^{31} \mathrm{P}$ NMR $\left(202.4 \mathrm{MHz}, \mathrm{C}_{5} \mathrm{D}_{5} \mathrm{~N}\right)$ Dp 49.3; HRMS calcd for $\mathrm{C}_{26} \mathrm{H}_{53} \mathrm{O}_{6} \mathrm{PNa}^{+}$: 515.3472. Found: 515.3504.

Diethyl 3,4,6-tri- $O$-benzyl-2,5-anhydroglucityl phosphonate (25). $R_{f} 0.26$ (2:1, EtOAc:hexane); $[\square]_{\mathrm{D}}^{25}+17.4\left(\mathrm{c} 1.0, \mathrm{CHCl}_{3}\right) ;{ }^{1} \mathrm{H}$ NMR (400 MHz, $\left.\mathrm{CD}_{3} \mathrm{OD}\right) \square_{\mathrm{H}} 1.23(\mathrm{t}, 3 \mathrm{H}, J$ = 7.1 Hz), $1.23(\mathrm{t}, 3 \mathrm{H}, J=7.0 \mathrm{~Hz}), 2.34 \square 2.14(\mathrm{~m}, 2 \mathrm{H}), 3.42(\mathrm{dd}, 1 \mathrm{H}, J=9.9,6.4 \mathrm{~Hz}), 3.51$ $(\mathrm{dd}, 1 \mathrm{H}, J=9.9,5.9 \mathrm{~Hz}), 3.93 \square 3.86(\mathrm{~m}, 2 \mathrm{H}), 4.07-3.95(\mathrm{~m}, 5 \mathrm{H}), 4.27 \square 4.19(\mathrm{~m}, 1 \mathrm{H})$,

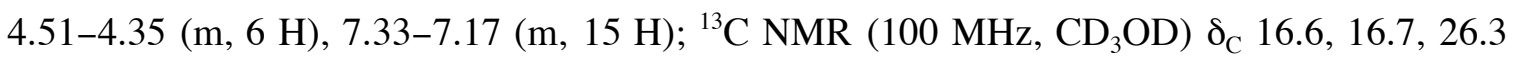
(d, $J=141.1 \mathrm{~Hz}), 63.2(\mathrm{~d}, J=6.3 \mathrm{~Hz}), 63.5(\mathrm{~d}, J=6.3 \mathrm{~Hz}), 71.6,72.6,72.7,74.3,77.4,84.3$, $84.4,84.4,128.8,128.9,129.0,129.1,129.1,129.5,129.5,129.6,139.4,139.4,139.6 ;{ }^{31} \mathrm{P}$ NMR (101.2 MHz, $\left.\mathrm{CDCl}_{3}\right) \square_{\mathrm{p}}$ 29.8; HRMS calcd for $\mathrm{C}_{31} \mathrm{H}_{39} \mathrm{O}_{7} \mathrm{PNa}^{+}$: 577.2326. Found: 577.2332 .

3,4,6-tri- $O$-benzyl-2,5-anhydroglucityl phosphine $(26) . \quad R_{f} 0.41 \quad(6: 1$, hexane:EtOAc); [ [ $]_{\mathrm{D}}+33.9\left(\right.$ c 1.0, $\left.\mathrm{CHCl}_{3}\right) ;{ }^{1} \mathrm{H}$ NMR $\left(400 \mathrm{MHz}, \mathrm{CDCl}_{3}\right) \square_{\mathrm{H}} 1.92(\mathrm{dd}, 2 \mathrm{H}$,

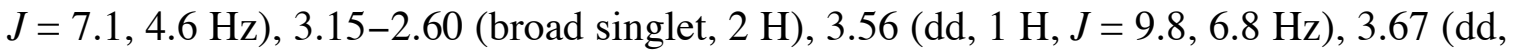
$1 \mathrm{H}, J=9.8,5.8 \mathrm{~Hz}), 4.01 \square 3.95(\mathrm{~m}, 2 \mathrm{H}), 4.19 \square 4.08(\mathrm{~m}, 2 \mathrm{H}), 4.42(\mathrm{~d}, 1 \mathrm{H}, J=11.7 \mathrm{~Hz})$, 4.67\4.54 (m, $5 \mathrm{H}), 7.28 \square 7.48(\mathrm{~m}, 15 \mathrm{H}) ;{ }^{13} \mathrm{C}$ NMR $\left(100 \mathrm{MHz}, \mathrm{CDCl}_{3}\right) \square_{\mathrm{c}} 13.0(\mathrm{~d}, J=$ $10.5 \mathrm{~Hz}), 70.5,71.3,71.4,73.3,82.2,82.8,83.2(\mathrm{~d}, J=1.3 \mathrm{~Hz}), 83.4,127.5,127.6$, 
$127.7,127.7,127.8,127.8,127.9,128.3,128.4,128.4,128.6,137.6,137.7,138.2 ;{ }^{31} \mathrm{P}$ NMR (161.9 MHz, $\left.\mathrm{CDCl}_{3}\right) \square_{p} \square 150.6$; HRMS calcd for $\mathrm{C}_{27} \mathrm{H}_{31} \mathrm{O}_{4} \mathrm{PNa}^{+}$: 473.1852. Found: 473.1820.

1-(Octyl)-3,4,6-tri- $O$-benzyl-2,5-anhydroglucityl phosphinic acid (28a). $R_{f}$ $0.42\left(6: 1, \mathrm{CH}_{2} \mathrm{Cl}_{2}: \mathrm{CH}_{3} \mathrm{OH}\right) ;[\square]_{\mathrm{D}}+11.7\left(c\right.$ 1.1, $\left.\mathrm{CHCl}_{3}\right) ;{ }^{1} \mathrm{H} \mathrm{NMR}\left(400 \mathrm{MHz}, \mathrm{C}_{5} \mathrm{D}_{5} \mathrm{~N}\right) \square_{\mathrm{H}}$ $0.84(\mathrm{t}, 3 \mathrm{H}, J=7.1 \mathrm{~Hz}), 1.40 \square 1.15(\mathrm{~m}, 10 \mathrm{H}), 2.07 \square 1.80(\mathrm{~m}, 4 \mathrm{H}), 2.69 \square 2.60(\mathrm{~m}, 2 \mathrm{H})$, $3.85 \square 3.75(\mathrm{~m}, 2 \mathrm{H}), 4.29 \square 4.26(\mathrm{~m}, 1 \mathrm{H}), 4.42 \square 4.32(\mathrm{~m}, 2 \mathrm{H}), 4.78 \square 4.53(\mathrm{~m}, 6 \mathrm{H})$, 4.98\4.89 (m, $1 \mathrm{H}), 7.50 \square 7.20$ (m, $15 \mathrm{H}), 12.27$ (br. s, $1 \mathrm{H}) ;{ }^{13} \mathrm{C}$ NMR (100 MHz, $\left.\mathrm{C}_{5} \mathrm{D}_{5} \mathrm{~N}\right) \square_{\mathrm{c}} 14.3,22.9,29.4,29.6,30.5(\mathrm{~d}, J=88.7 \mathrm{~Hz}), 31.4(\mathrm{~d}, J=14.5 \mathrm{~Hz}), 31.5(\mathrm{~d}, J=$ $94.0 \mathrm{~Hz}), 32.1,71.6,71.6,71.9,73.4,77.6,83.4,84.2(\mathrm{~d}, J=6.0 \mathrm{~Hz}), 84.7,127.9,128.0$, $128.1,128.1,128.3,128.7,128.8,138.8,139.0,139.1 ;{ }^{31} \mathrm{P} N \mathrm{NMR}\left(161.9 \mathrm{MHz}, \mathrm{C}_{5} \mathrm{D}_{5} \mathrm{~N}\right) \square_{p}$ 48.6; HRMS calcd for $\mathrm{C}_{35} \mathrm{H}_{47} \mathrm{O}_{6} \mathrm{PNa}^{+}$: 617.3002. Found: 617.3007.

1-(Decyl)-3,4,6-tri- $O$-benzyl-2,5-anhydroglucityl phosphinic acid $(28 \mathrm{~b}) . \quad R_{f}$ $0.57\left(3: 1, \mathrm{CH}_{2} \mathrm{Cl}_{2}: \mathrm{CH}_{3} \mathrm{OH}\right) ;[\square]_{\mathrm{D}}+12.4\left(c 1.0, \mathrm{CHCl}_{3}\right) ;{ }^{1} \mathrm{H} \mathrm{NMR}\left(500 \mathrm{MHz}, \mathrm{C}_{5} \mathrm{D}_{5} \mathrm{~N}\right) \square_{\mathrm{H}}$ $0.85(\mathrm{t}, 3 \mathrm{H}, J=7.1 \mathrm{~Hz}), 1.41 \square 1.15(\mathrm{~m}, 14 \mathrm{H}), 2.08 \square 1.81(\mathrm{~m}, 4 \mathrm{H}), 2.68 \square 2.60(\mathrm{~m}, 2 \mathrm{H})$, $3.83 \square 3.73(\mathrm{~m}, 2 \mathrm{H}), 4.27 \square 4.24(\mathrm{~m}, 1 \mathrm{H}), 4.39 \square 4.31(\mathrm{~m}, 2 \mathrm{H}), 4.74 \square 4.52(\mathrm{~m}, 6 \mathrm{H})$,

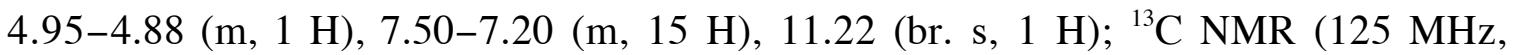
$\left.\mathrm{C}_{5} \mathrm{D}_{5} \mathrm{~N}\right) \square_{\mathrm{c}} 14.3,22.9,29.6,29.7,29.8,29.9,30.5(\mathrm{~d}, J=88.5 \mathrm{~Hz}), 31.4(\mathrm{~d}, J=14.6 \mathrm{~Hz})$, $31.6(\mathrm{~d}, J=92.7 \mathrm{~Hz}), 32.1,71.6,71.6,71.9,73.4,77.6,83.4,84.2(\mathrm{~d}, J=6.0 \mathrm{~Hz}), 84.7$, $127.9,128.0,128.1,128.1,128.3,128.7,128.8,138.8,139.0,139.1 ;{ }^{31} \mathrm{P}$ NMR (161.9 $\left.\mathrm{MHz}, \mathrm{C}_{5} \mathrm{D}_{5} \mathrm{~N}\right) \square, 49.0$; HRMS calcd for $\mathrm{C}_{37} \mathrm{H}_{51} \mathrm{O}_{6} \mathrm{PNa}^{+}: 645.3315$. Found: 645.3344.

1-(Dodecyl)-3,4,6-tri- $O$-benzyl-2,5-anhydroglucityl phosphinic acid (28c). $R_{f}$ $0.27\left(6: 1, \mathrm{CH}_{2} \mathrm{Cl}_{2}: \mathrm{CH}_{3} \mathrm{OH}\right) ;[\square]_{\mathrm{D}}+11.4\left(c \quad 1.1, \mathrm{CHCl}_{3}\right) ;{ }^{1} \mathrm{H} \mathrm{NMR}\left(500 \mathrm{MHz}, \mathrm{C}_{5} \mathrm{D}_{5} \mathrm{~N}\right) \square_{\mathrm{H}}$ 
$0.87(\mathrm{t}, 3 \mathrm{H}, J=7.1 \mathrm{~Hz}), 1.45 \square 1.18(\mathrm{~m}, 18 \mathrm{H}), 2.10 \square 1.82(\mathrm{~m}, 4 \mathrm{H}), 2.69 \square 2.60(\mathrm{~m}, 2 \mathrm{H})$,

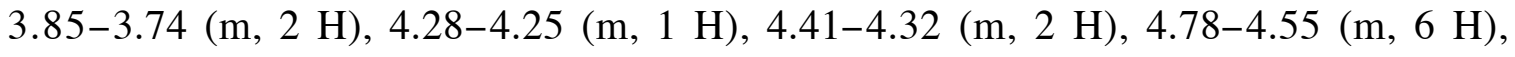
4.97\4.89 (m, $1 \mathrm{H}), 7.50 \square 7.25$ (m, $15 \mathrm{H}), 8.05$ (br. s, $1 \mathrm{H}) ;{ }^{13} \mathrm{C} \mathrm{NMR}\left(125 \mathrm{MHz}, \mathrm{C}_{5} \mathrm{D}_{5} \mathrm{~N}\right)$ $\square_{\mathrm{c}} 14.3,23.0,29.6,29.7,29.8,29.9,30.0,30.2,30.6(\mathrm{~d}, J=89.2 \mathrm{~Hz}), 31.4(\mathrm{~d}, J=14.4$ $\mathrm{Hz}), 31.6(\mathrm{~d}, J=92.2 \mathrm{~Hz}), 32.2,71.6,71.6,71.9,73.4,77.6,83.4,84.2(\mathrm{~d}, J=5.4 \mathrm{~Hz})$, $84.7,127.9,128.0,128.1,128.1,128.3,128.7,128.8,128.8,138.8,139.0,139.1 ;{ }^{31} \mathrm{P}$ NMR (161.9 MHz, $\left.\mathrm{C}_{5} \mathrm{D}_{5} \mathrm{~N}\right)$ \, 48.8; HRMS calcd for $\mathrm{C}_{39} \mathrm{H}_{55} \mathrm{O}_{6} \mathrm{PNa}^{+}:$673.3628. Found: 673.3622.

1-(Hexadecyl)-3,4,6-tri-O-benzyl-2,5-anhydroglucityl phosphinic acid (28d).

$R_{f} 0.41\left(6: 1, \mathrm{CH}_{2} \mathrm{Cl}_{2}: \mathrm{CH}_{3} \mathrm{OH}\right) ;[\square]_{\mathrm{D}}+10.3\left(c 1.0, \mathrm{CHCl}_{3}\right) ;{ }^{1} \mathrm{H} \mathrm{NMR}\left(500 \mathrm{MHz}, \mathrm{C}_{5} \mathrm{D}_{5} \mathrm{~N}\right) \square_{\mathrm{H}}$ $0.86(\mathrm{t}, 3 \mathrm{H}, J=7.1 \mathrm{~Hz}), 1.42 \square 1.18(\mathrm{~m}, 26 \mathrm{H}), 2.07 \square 1.81(\mathrm{~m}, 4 \mathrm{H}), 2.68 \square 2.60(\mathrm{~m}, 2 \mathrm{H})$, $3.83 \square 3.73(\mathrm{~m}, 2 \mathrm{H}), 4.26 \square 4.25(\mathrm{~m}, 1 \mathrm{H}), 4.39 \square 4.32(\mathrm{~m}, 2 \mathrm{H}), 4.74 \square 4.53(\mathrm{~m}, 6 \mathrm{H})$,

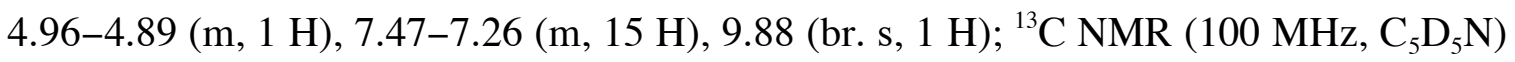
Z $14.3,23.0,29.7,29.7,29.7,29.9,30.0,30.0,30.5(\mathrm{~d}, J=88.5 \mathrm{~Hz}), 31.0,31.4(\mathrm{~d}, J=$ $14.5 \mathrm{~Hz}), 31.6(\mathrm{~d}, J=92.7 \mathrm{~Hz}), 32.2,71.3,71.6,71.9,73.4,77.6,83.4,84.2(\mathrm{~d}, J=5.7$ $\mathrm{Hz}), 84.7,127.9,128.0,128.1,128.1,128.3,128.7,128.8,138.8,139.0,139.1 ;{ }^{31} \mathrm{P}$ NMR (161.9 MHz, $\left.\mathrm{C}_{5} \mathrm{D}_{5} \mathrm{~N}\right) \square_{\mathrm{p}} 48.8$; HRMS calcd for $\mathrm{C}_{43} \mathrm{H}_{63} \mathrm{O}_{6} \mathrm{PNa}^{+}:$729.4254. Found: 729.4297.

1-(Eicosanyl)-3,4,6-tri- $O$-benzyl-2,5-anhydroglucityl phosphinic acid (28e). $R_{f} 0.29\left(6: 1, \mathrm{CH}_{2} \mathrm{Cl}_{2}: \mathrm{CH}_{3} \mathrm{OH}\right) ;[\square]_{\mathrm{D}}+9.7\left(c 1.1, \mathrm{CHCl}_{3}\right) ;{ }^{1} \mathrm{H} \mathrm{NMR}\left(500 \mathrm{MHz}, \mathrm{C}_{5} \mathrm{D}_{5} \mathrm{~N}\right) \square_{\mathrm{H}}$ $0.87(\mathrm{t}, 3 \mathrm{H}, J=7.1 \mathrm{~Hz}), 1.45 \square 1.17(\mathrm{~m}, 34 \mathrm{H}), 2.10 \square 1.81(\mathrm{~m}, 4 \mathrm{H}), 2.70 \square 2.59(\mathrm{~m}, 2 \mathrm{H})$,

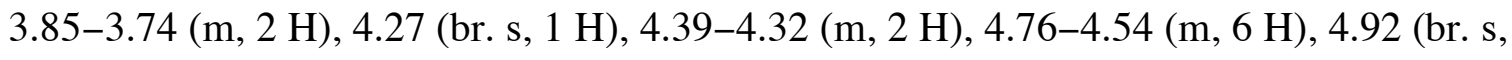
$1 \mathrm{H}), 7.50 \square 7.26$ (m, $15 \mathrm{H}), 8.10$ (br. s, $1 \mathrm{H}) ;{ }^{13} \mathrm{C} \mathrm{NMR}\left(100 \mathrm{MHz}, \mathrm{C}_{5} \mathrm{D}_{5} \mathrm{~N}\right) \square_{\mathrm{c}} 14.3,23.0$, 
29.6, 29.8, 30.0, 30.0, 30.1, $30.5(\mathrm{~d}, J=87.0 \mathrm{~Hz}), 30.9,31.5(\mathrm{~d}, J=12.5 \mathrm{~Hz}), 31.6(\mathrm{~d}, J=$ 89.6 Hz), 32.2, 71.6, 71.6, 71.9, 73.4, 77.7, 83.3, 84.2, 84.7, 127.9, 128.0, 128.1, 128.1, 128.3, 128.7, 128.8, 138.8, 139.0, 139.4; ${ }^{31}$ P NMR (202.4 MHz, $\left.\mathrm{C}_{5} \mathrm{D}_{5} \mathrm{~N}\right) \square_{\mathrm{p}} 47.4$; HRMS calcd for $\mathrm{C}_{47} \mathrm{H}_{71} \mathrm{O}_{6} \mathrm{PNa}^{+}:$785.4880. Found: 785.4893.

1-p-toluenesulfonyloxy-eicosane (32). $R_{f} 0.49$ (10:1, hexane:EtOAc); ${ }^{1} \mathrm{H}$ NMR $\left(250 \mathrm{MHz}, \mathrm{CDCl}_{3}\right) \square_{\mathrm{H}} 0.86(\mathrm{t}, 3 \mathrm{H}, J=6.9 \mathrm{~Hz}), 1.33 \square 1.14(\mathrm{~m} 34 \mathrm{H}), 1.68 \square 1.55(\mathrm{~m}, 2 \mathrm{H})$, $2.43(\mathrm{~s}, 3 \mathrm{H}), 4.00(\mathrm{t}, 2 \mathrm{H}, J=6.6 \mathrm{~Hz}), 7.31(\mathrm{~d}, 2 \mathrm{H}, J=8.0 \mathrm{~Hz}), 7.77(\mathrm{~d}, 2 \mathrm{H}, J=8.3 \mathrm{~Hz}) ;{ }^{13} \mathrm{C}$ $\operatorname{NMR}\left(62.5 \mathrm{MHz}, \mathrm{CDCl}_{3}\right) \square_{\mathrm{c}} 14.1,21.6,22.7,25.3,28.8,28.9,29.3,29.4,29.5,29.6,29.7$, 29.7, 31.9, 70.7, 127.9, 129.8, 133.3, 144.6; HRMS calcd for $\mathrm{C}_{27} \mathrm{H}_{48} \mathrm{O}_{3} \mathrm{SNa}^{+}$: 475.3216 . Found: 475.3240 . 

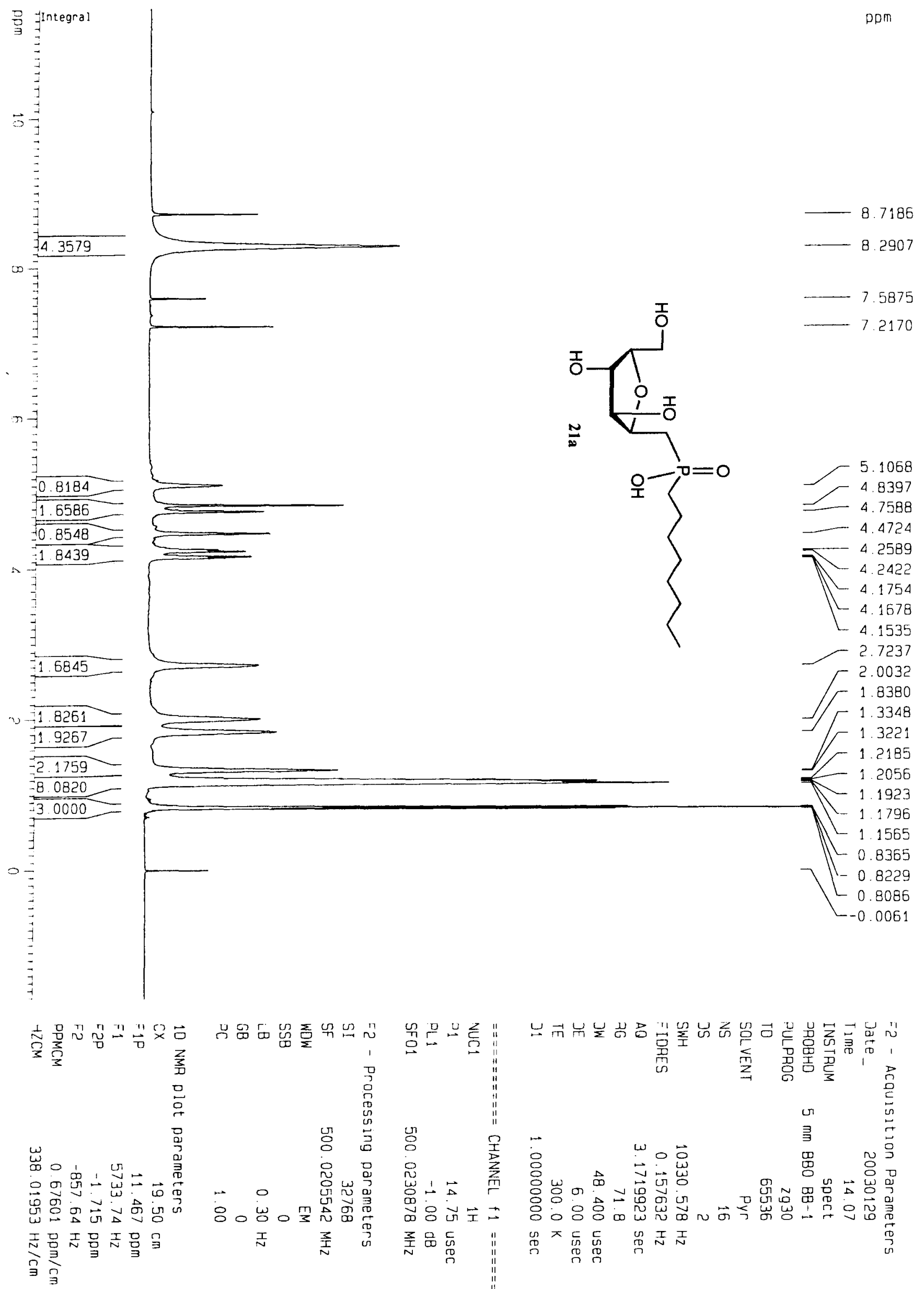

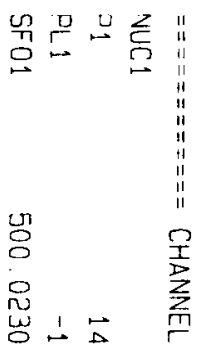

品:

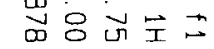
㴅品点

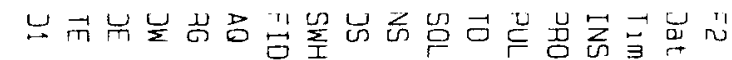

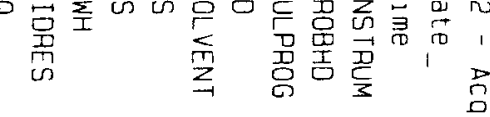

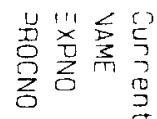

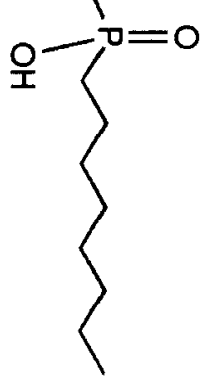

5.1068

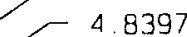

- 4.4724

7. 4.2589

$-4.2422$

$-4.1754$

4.1578

L 4.1535

$-2.7237$

$\Gamma .0032$

$\int 1.8380$

$\int \Gamma^{1.3348}$

$-1.2185$

$-1.2056$

- 1.1923

$-1.1796$

L 0.8365

- 0.8229

L 0.8086

$-0.0061$ 


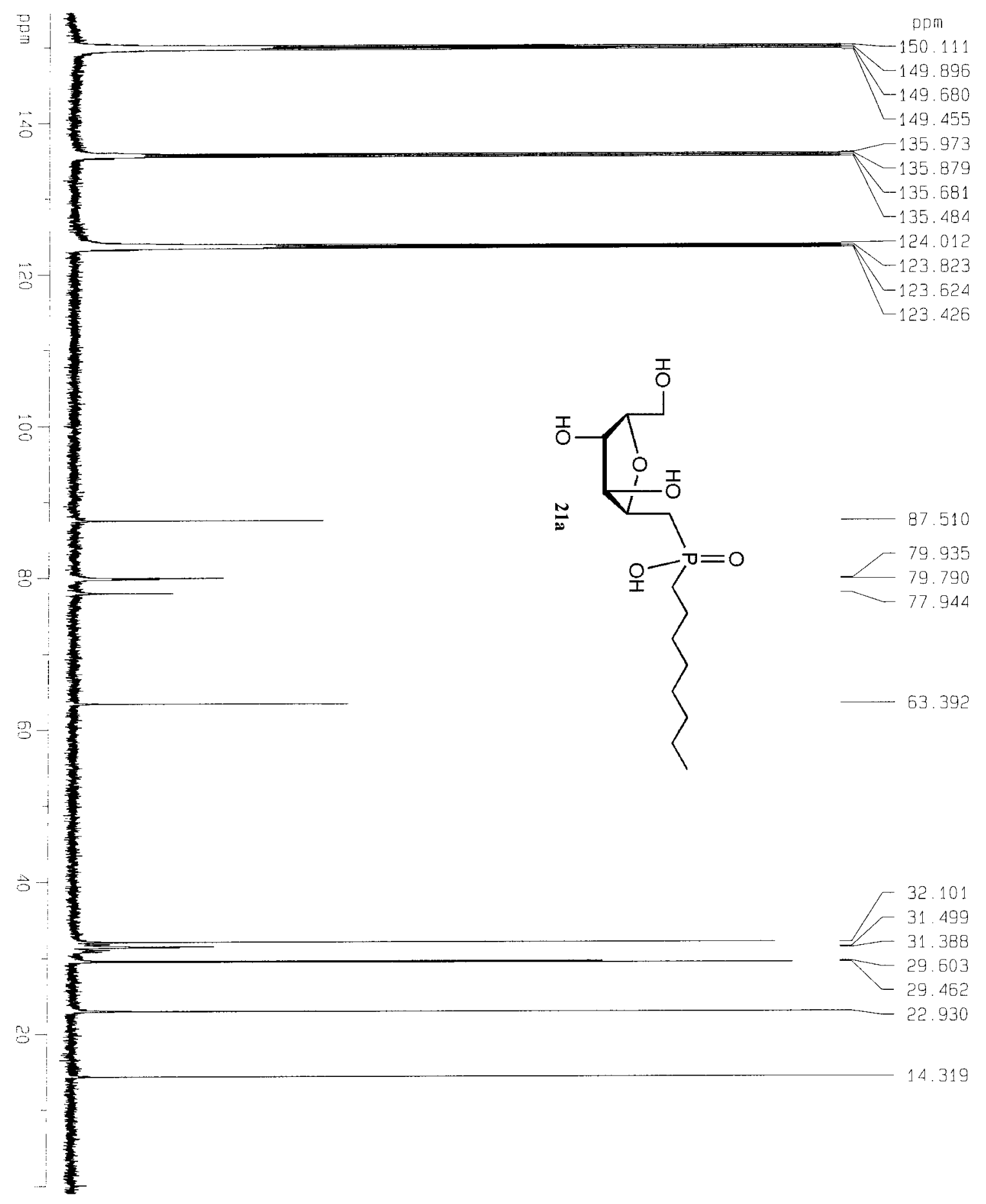

. 


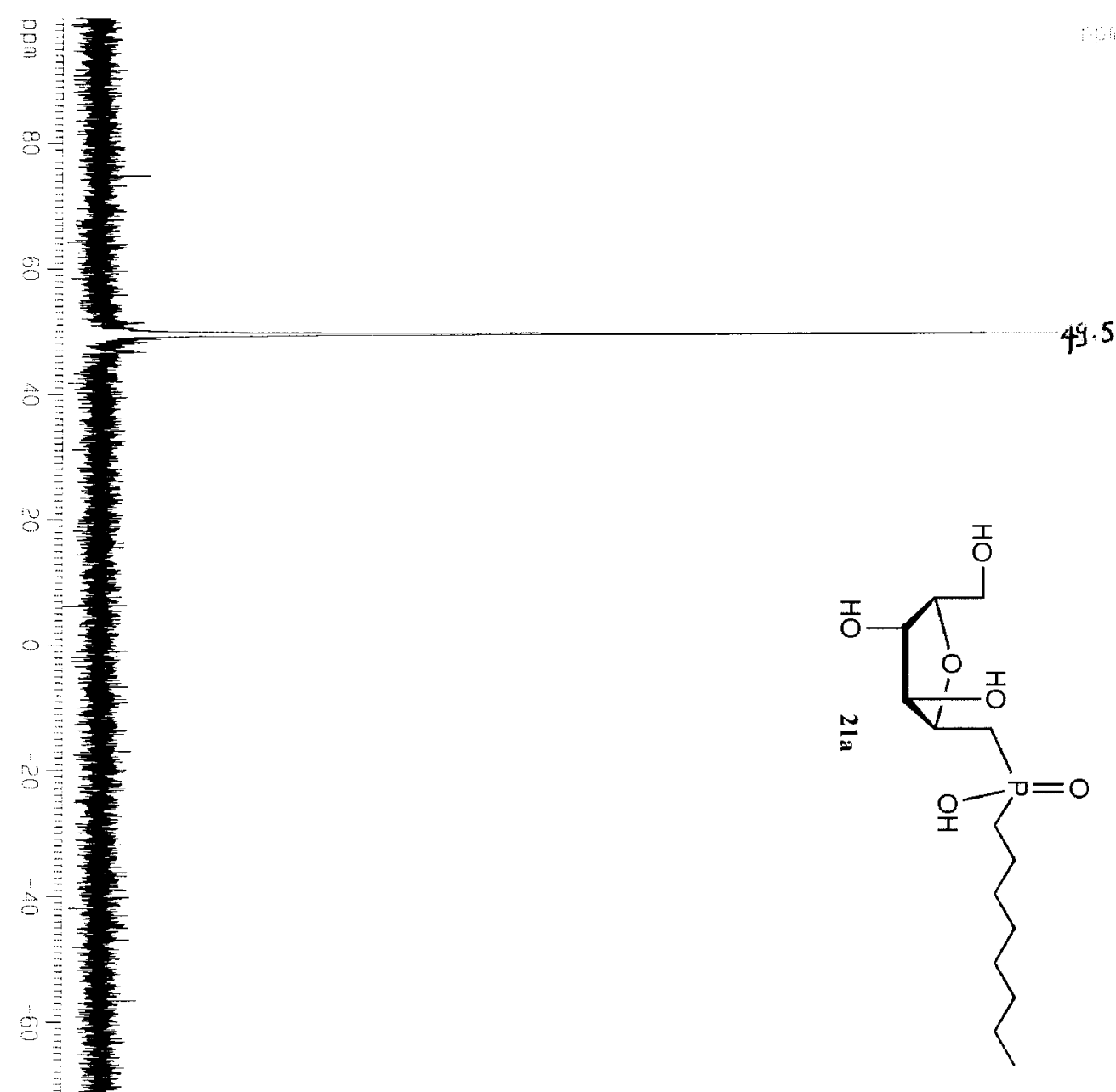

9.5744

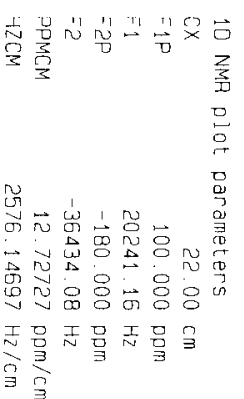
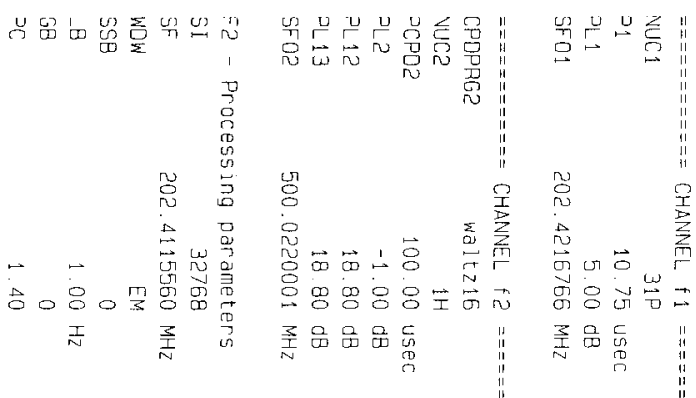

总名 


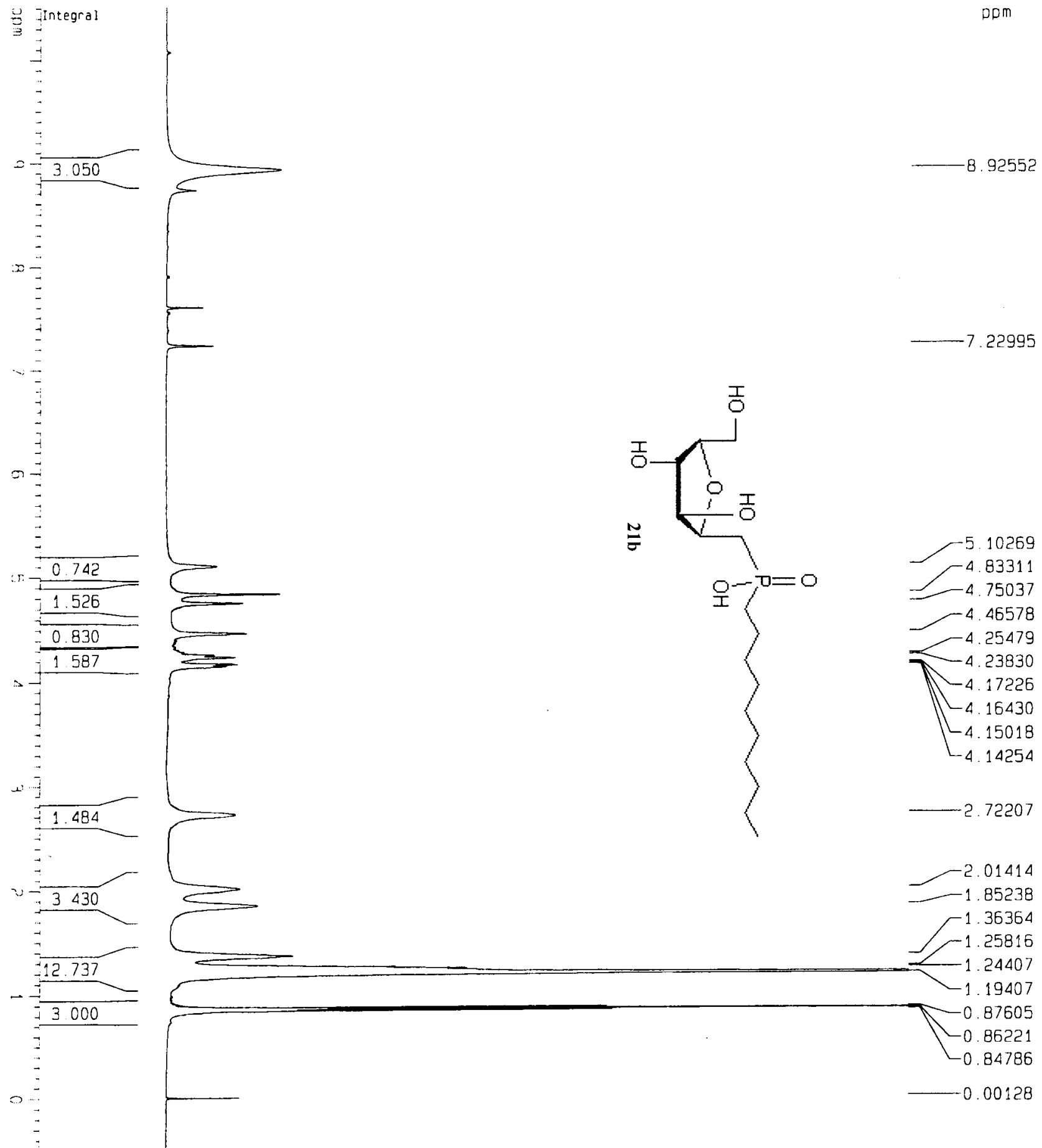

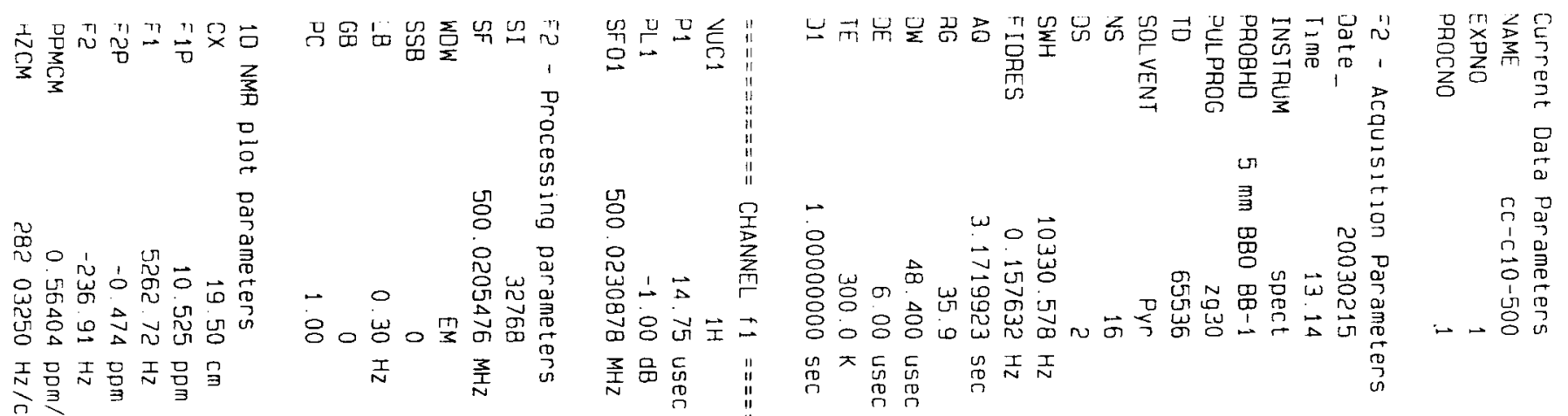



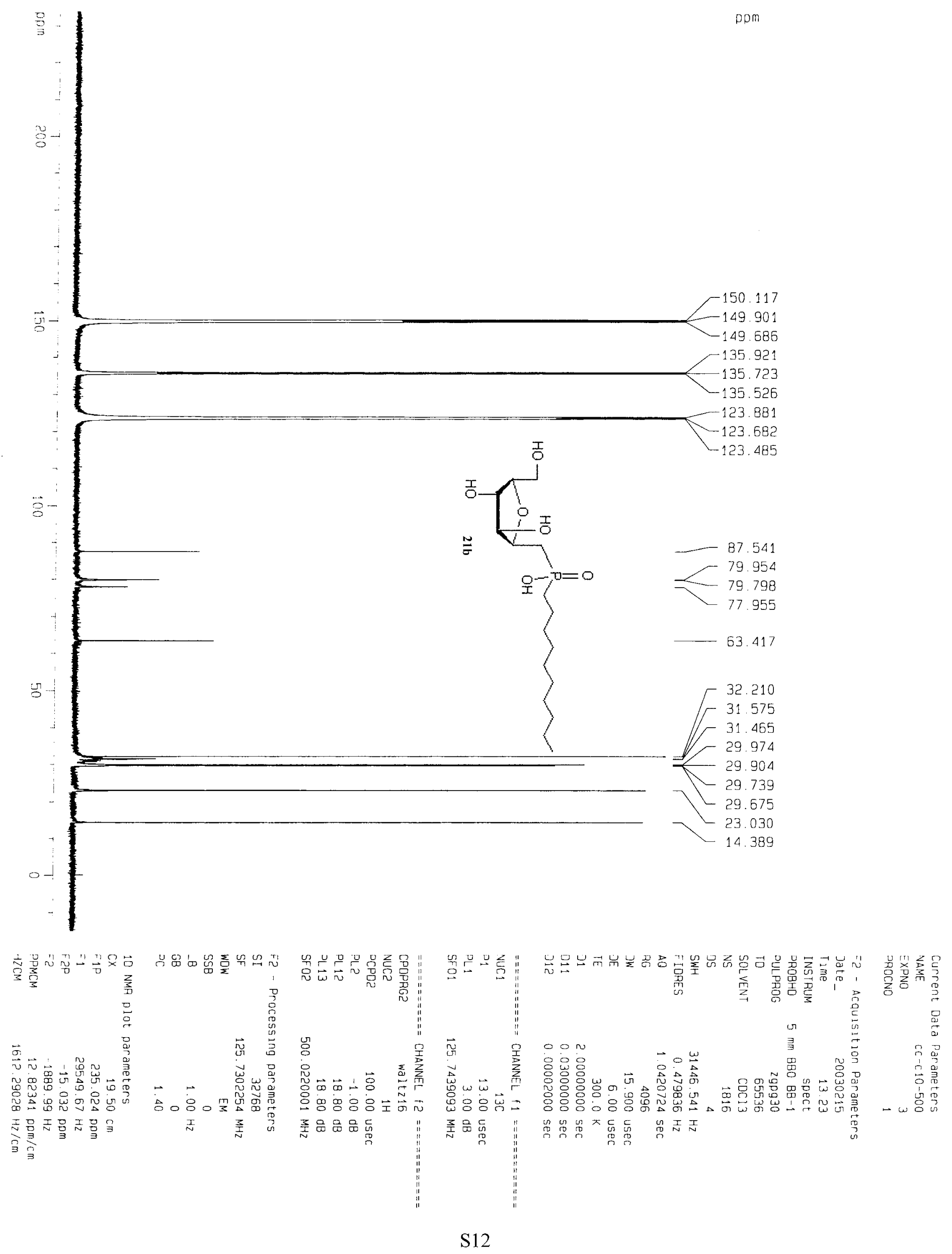


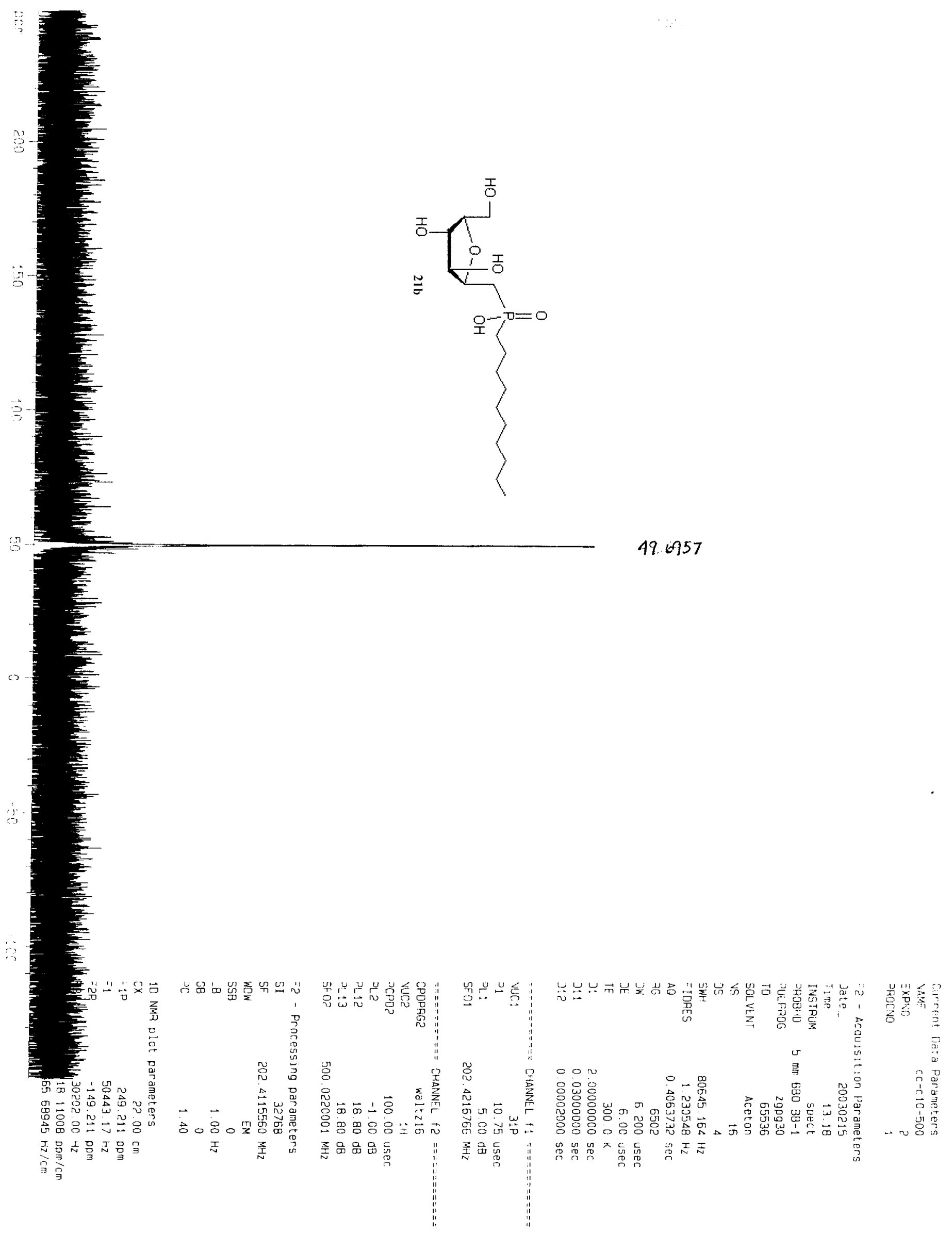




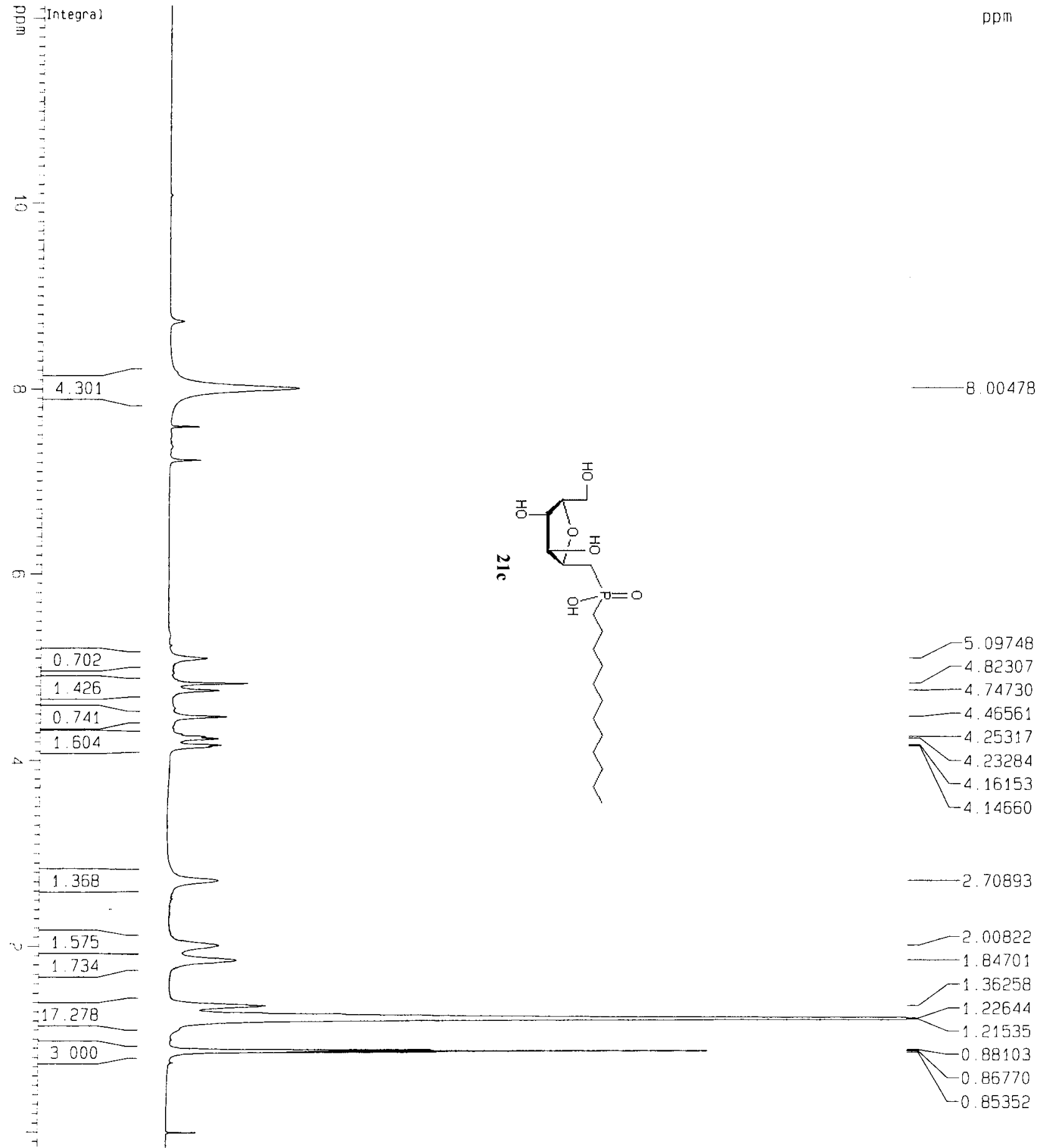

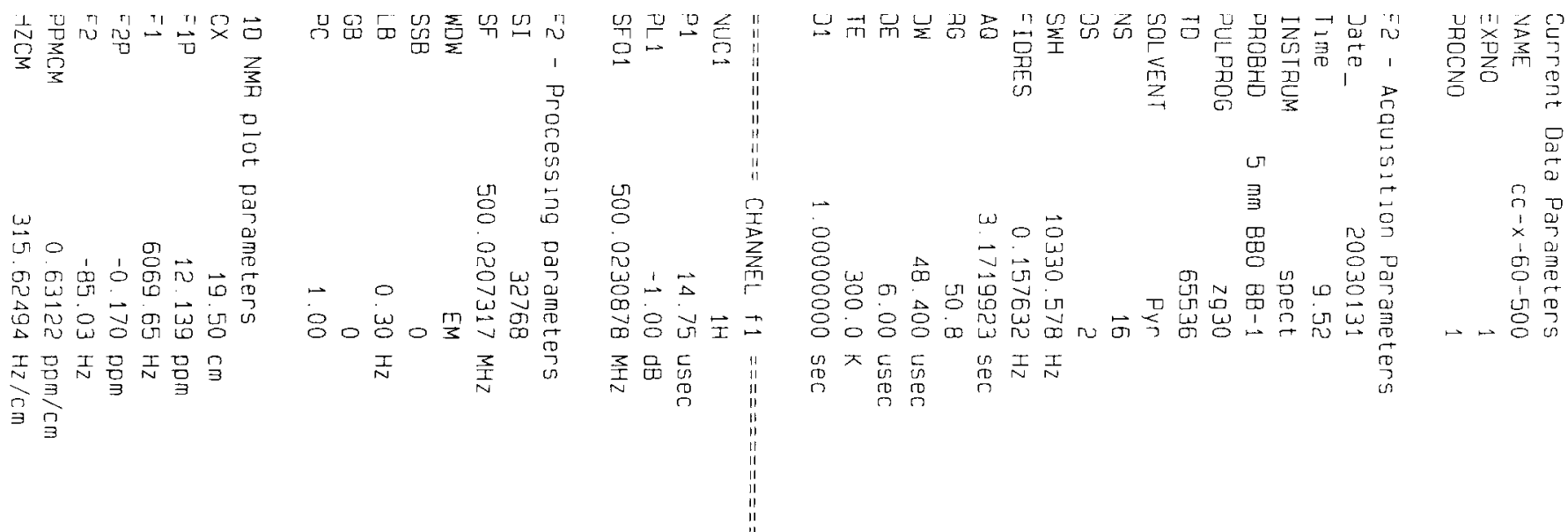



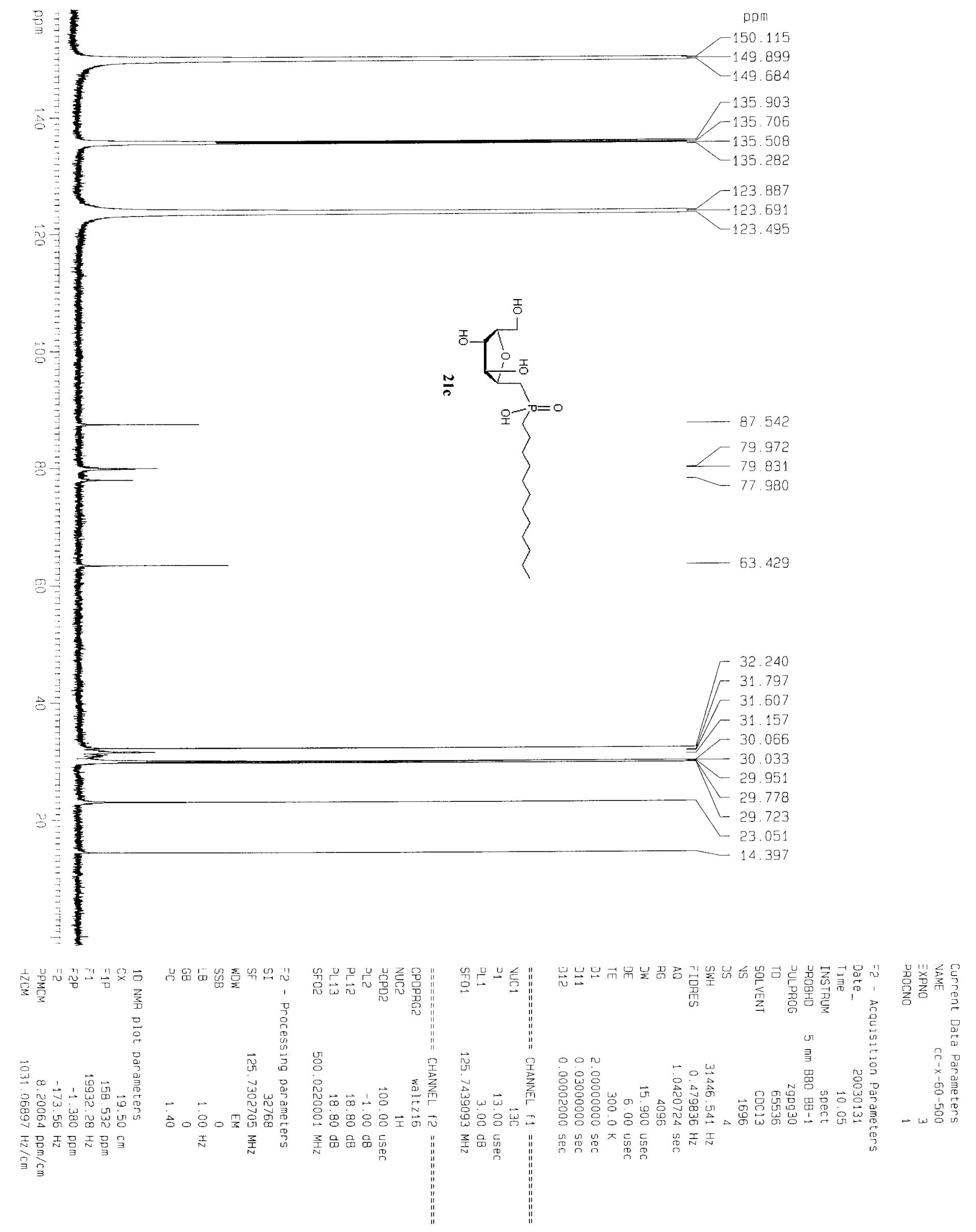


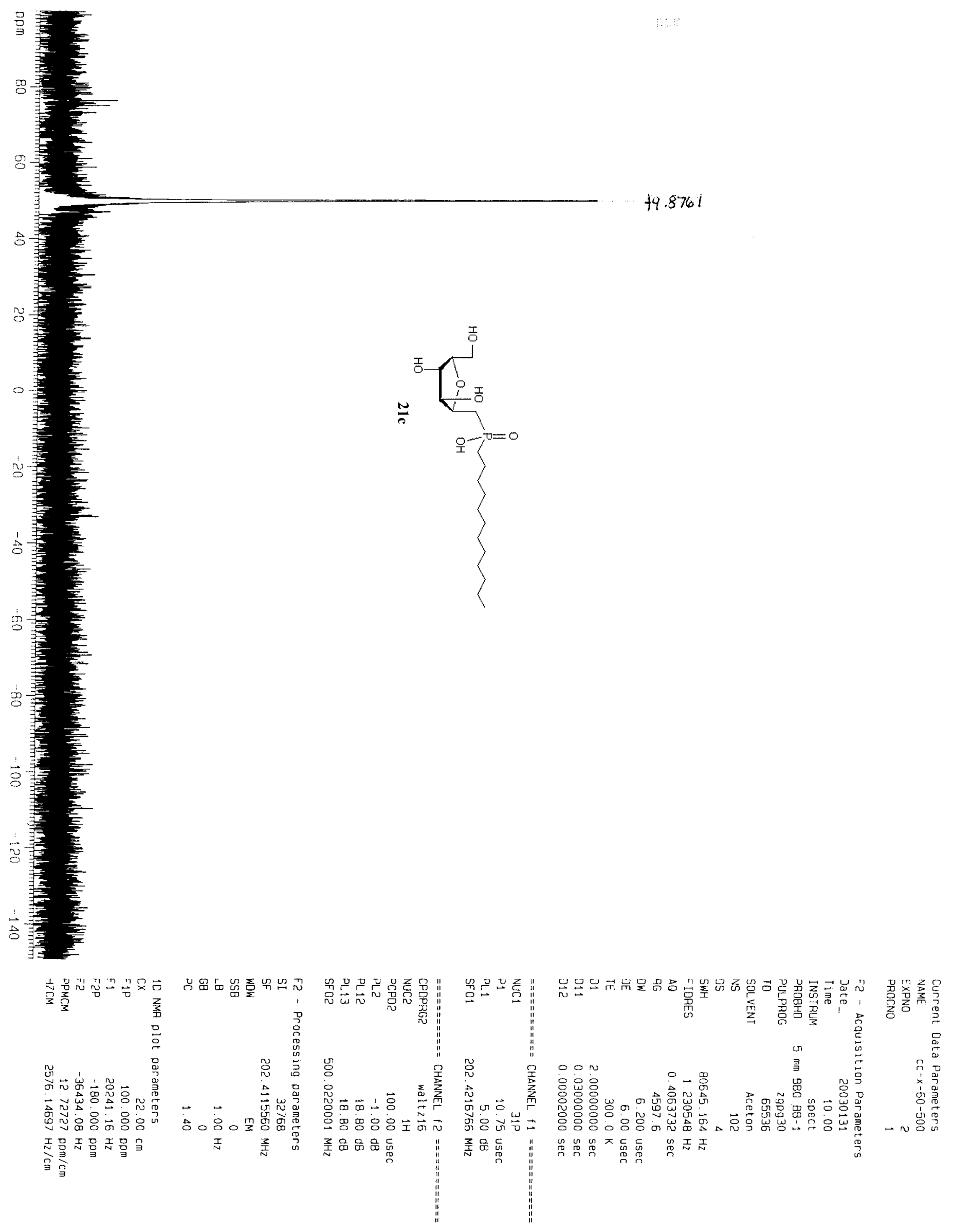




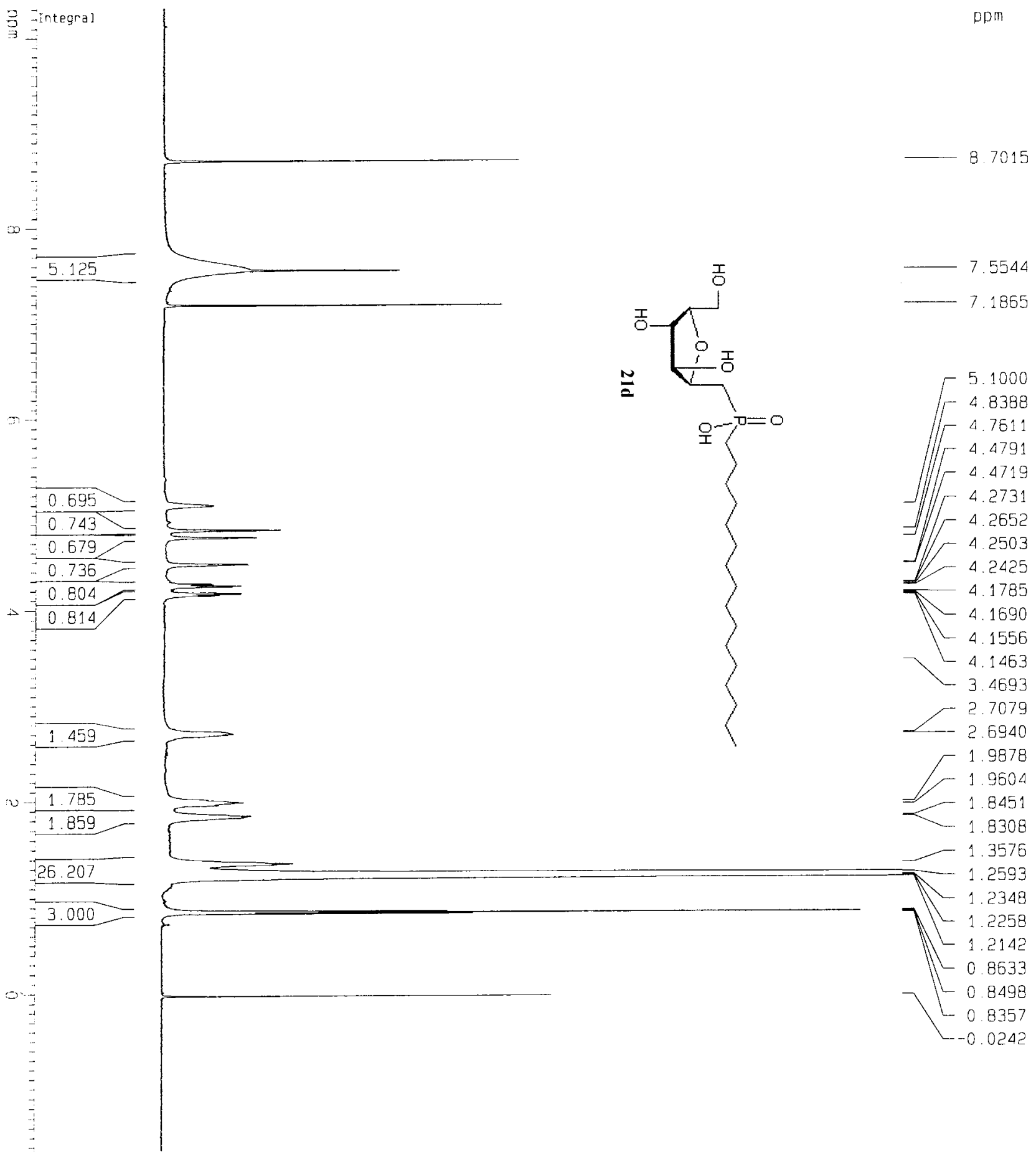

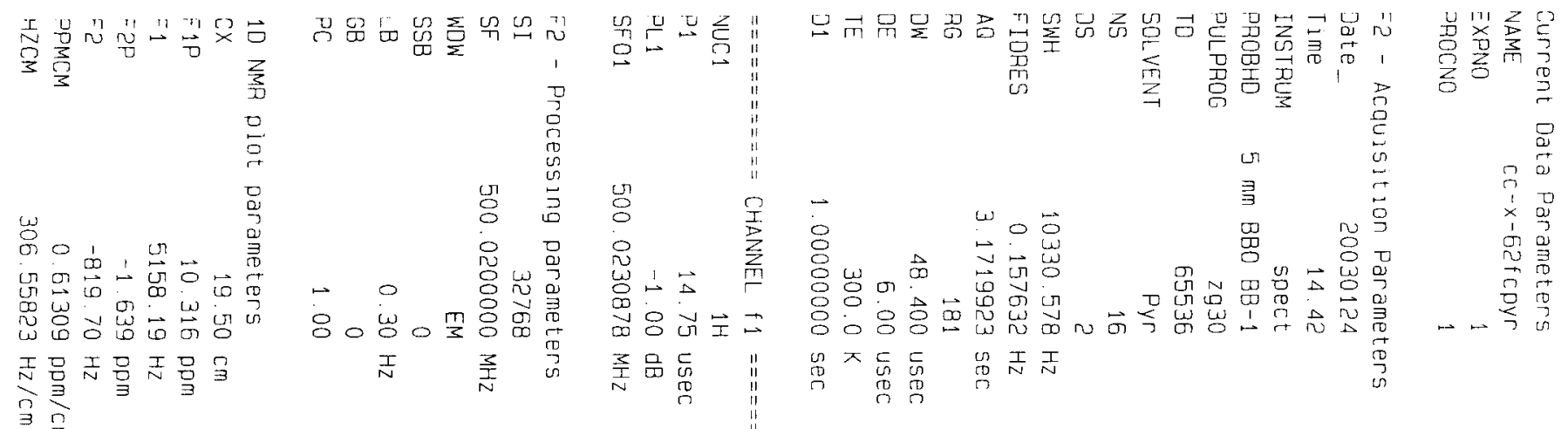




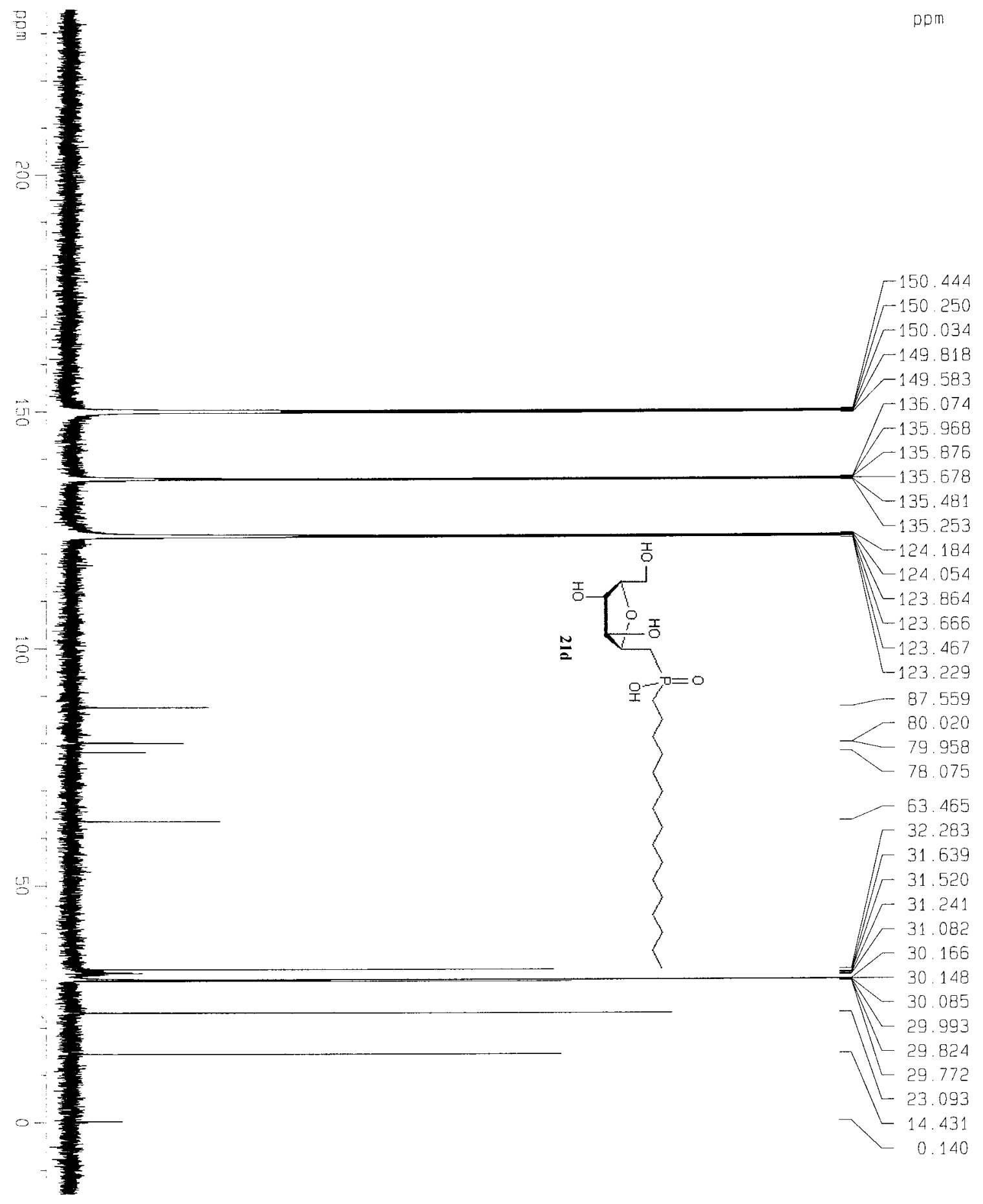

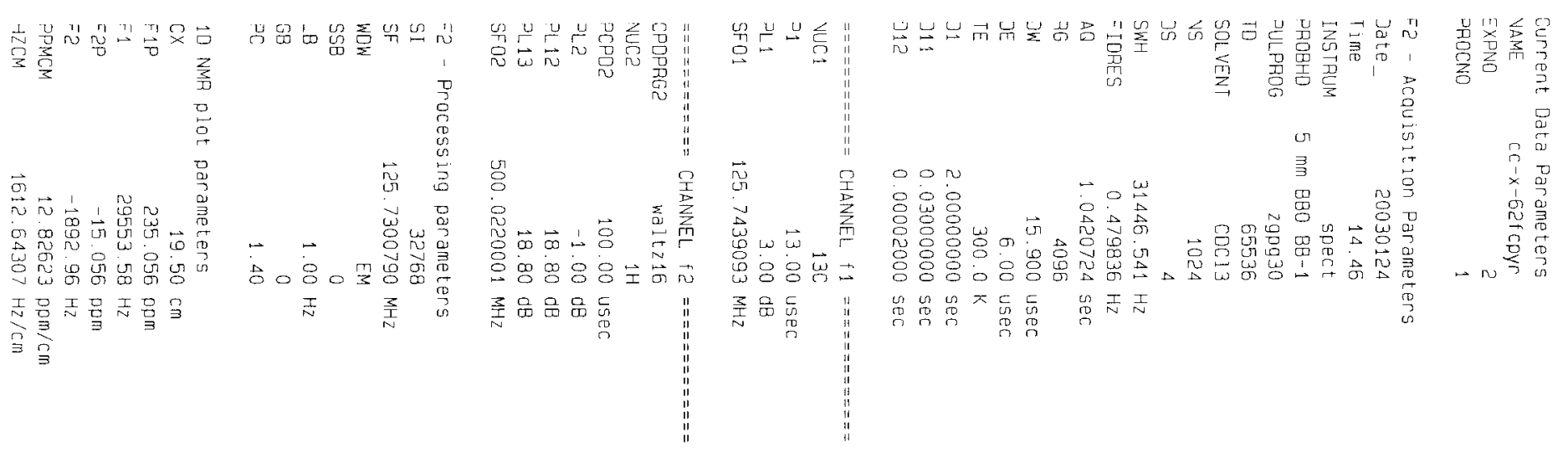



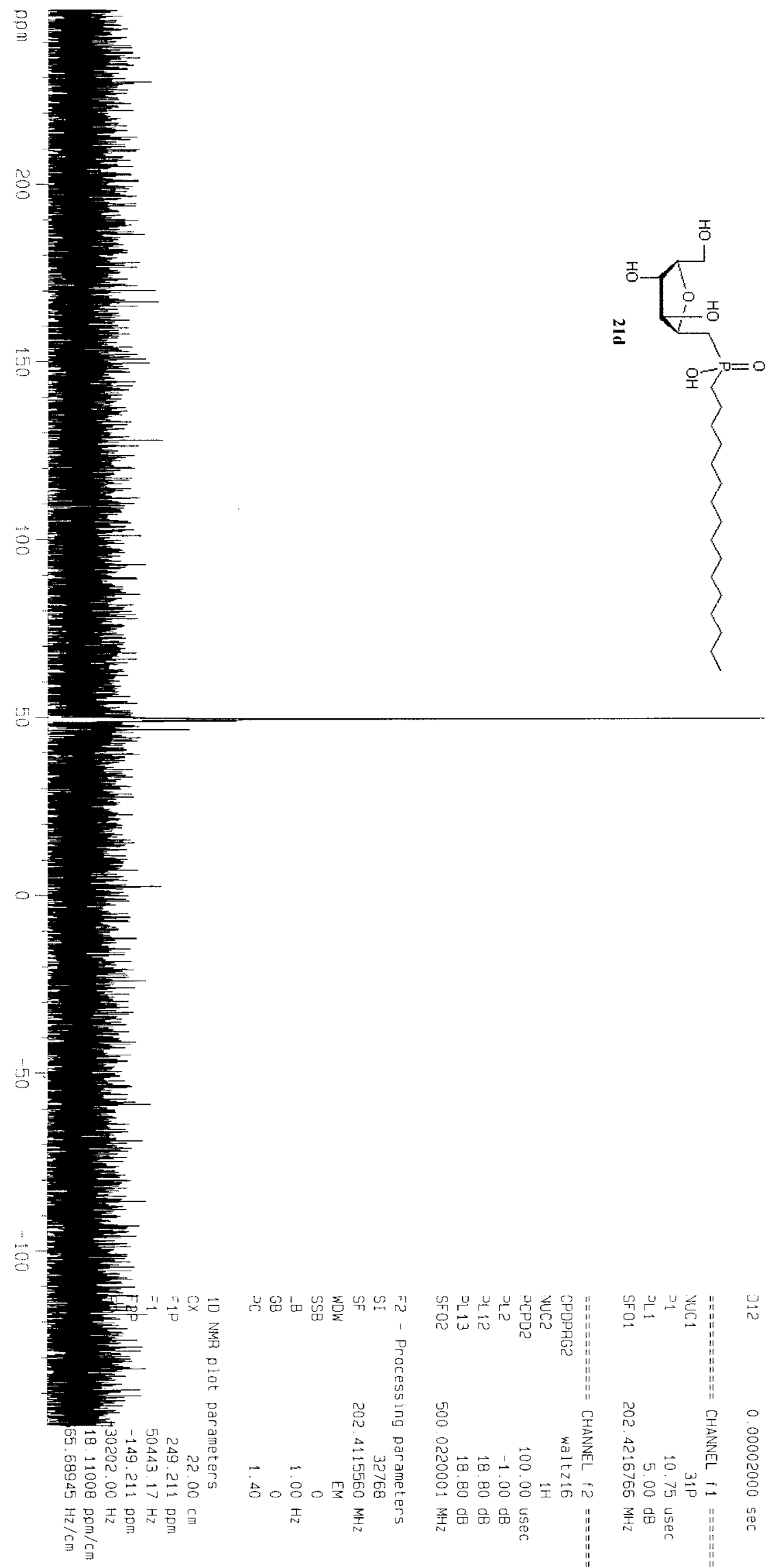

49. 4399

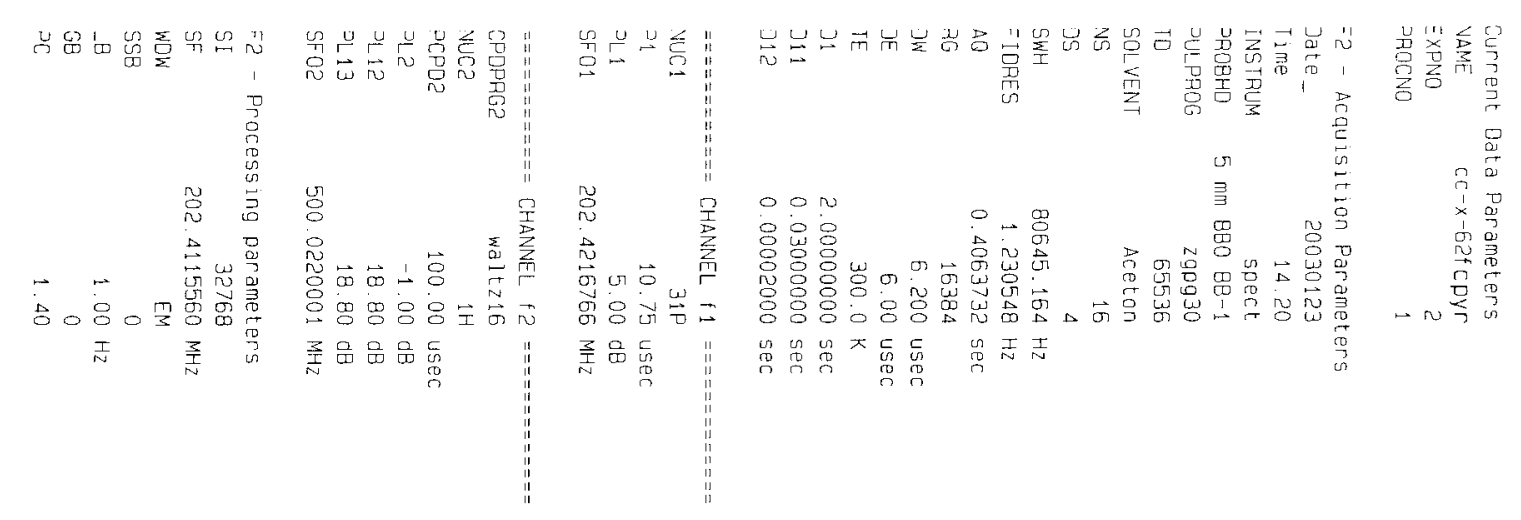




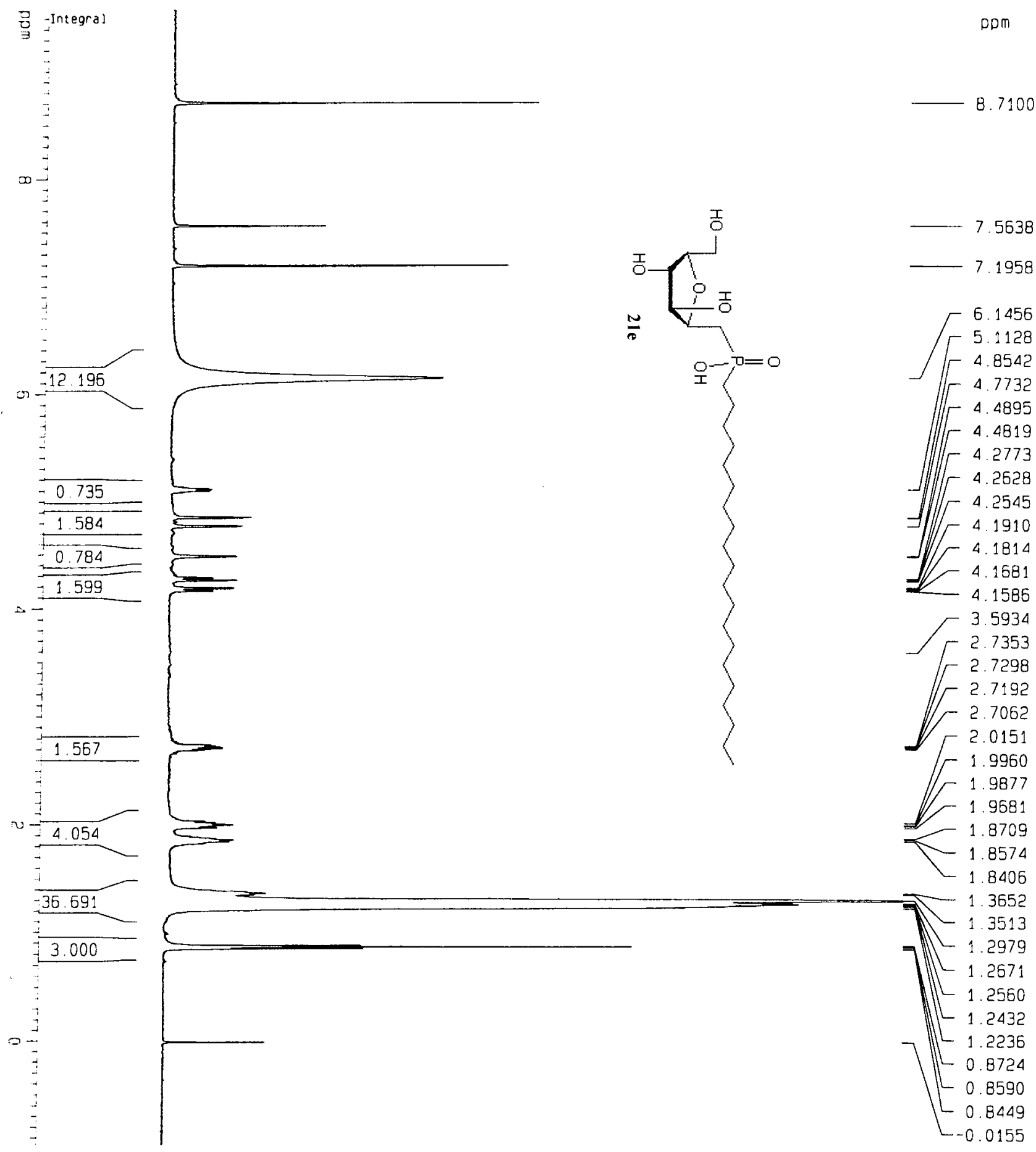

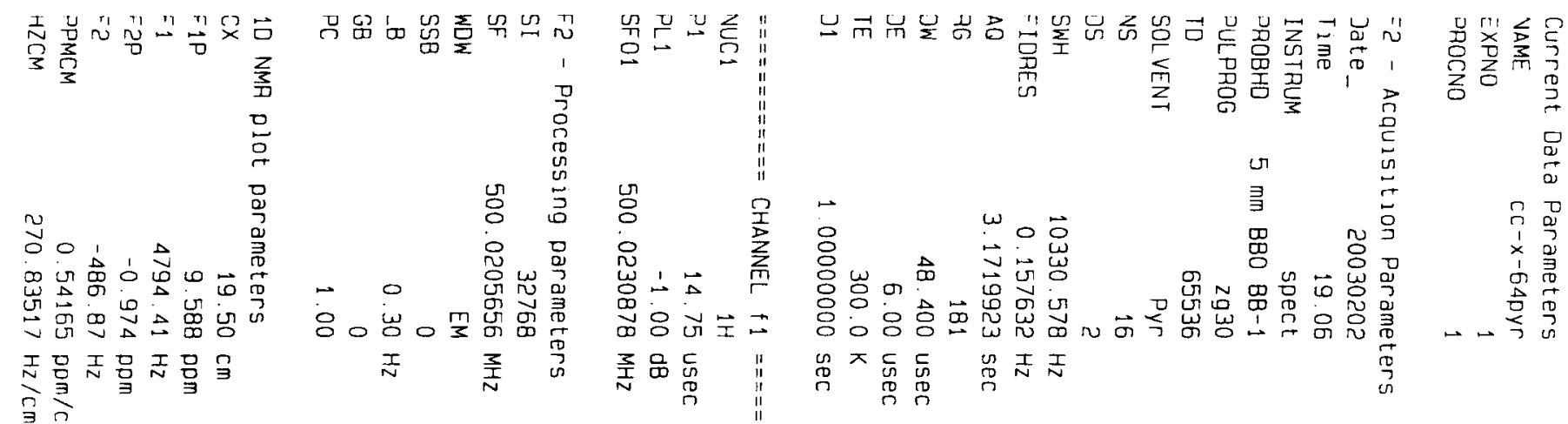




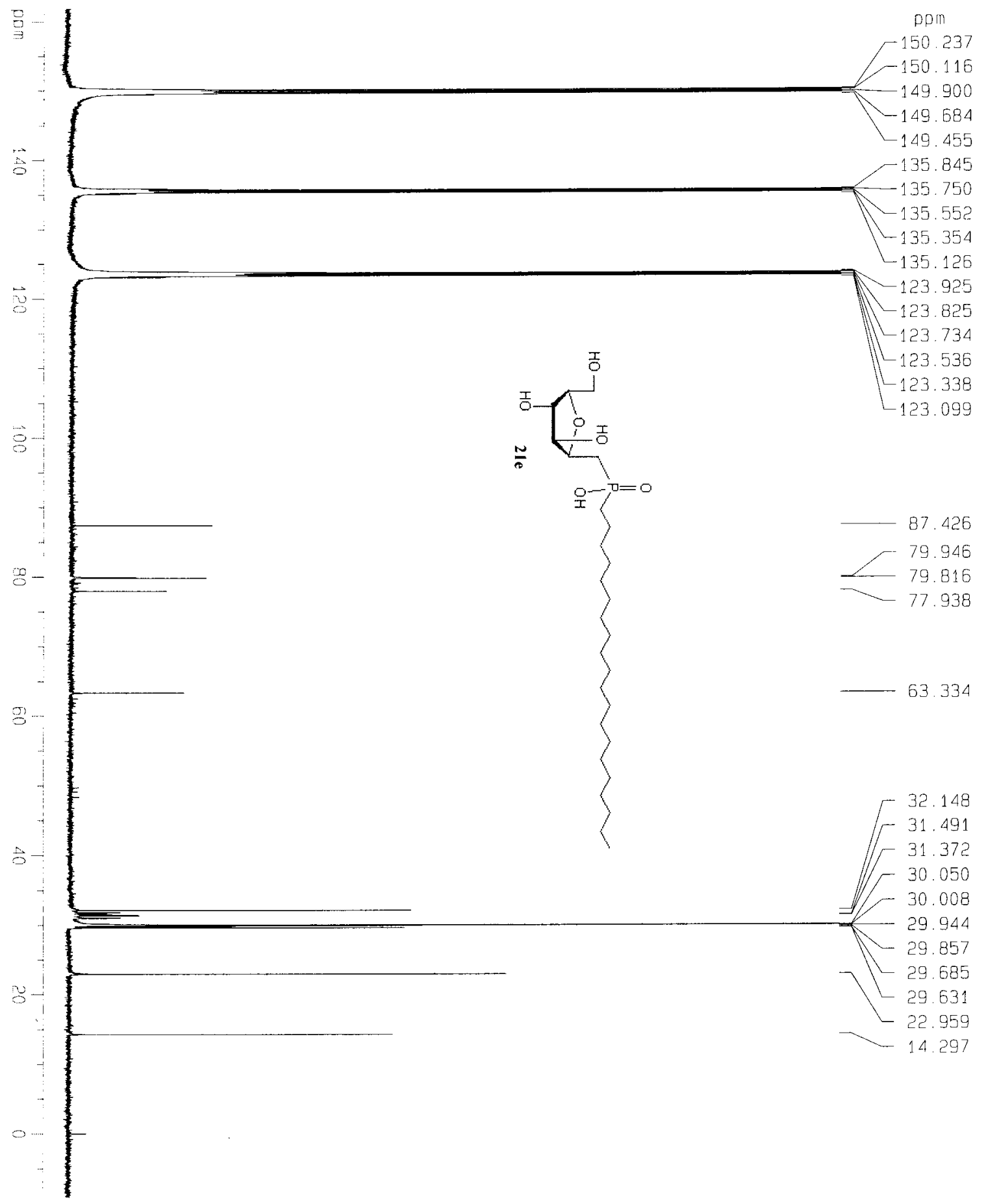

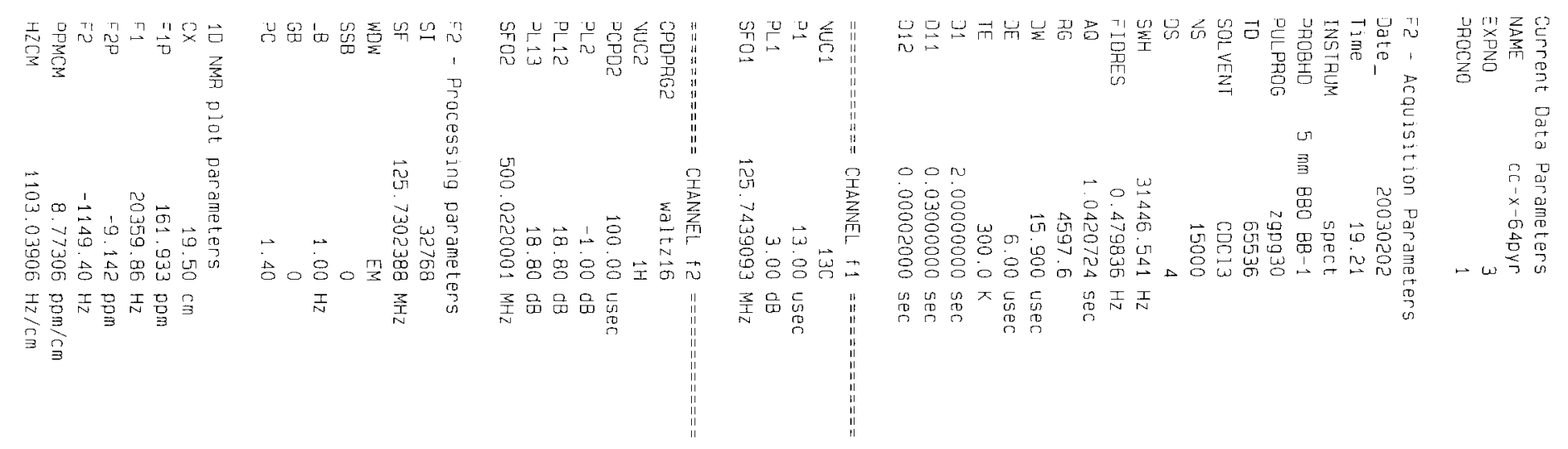



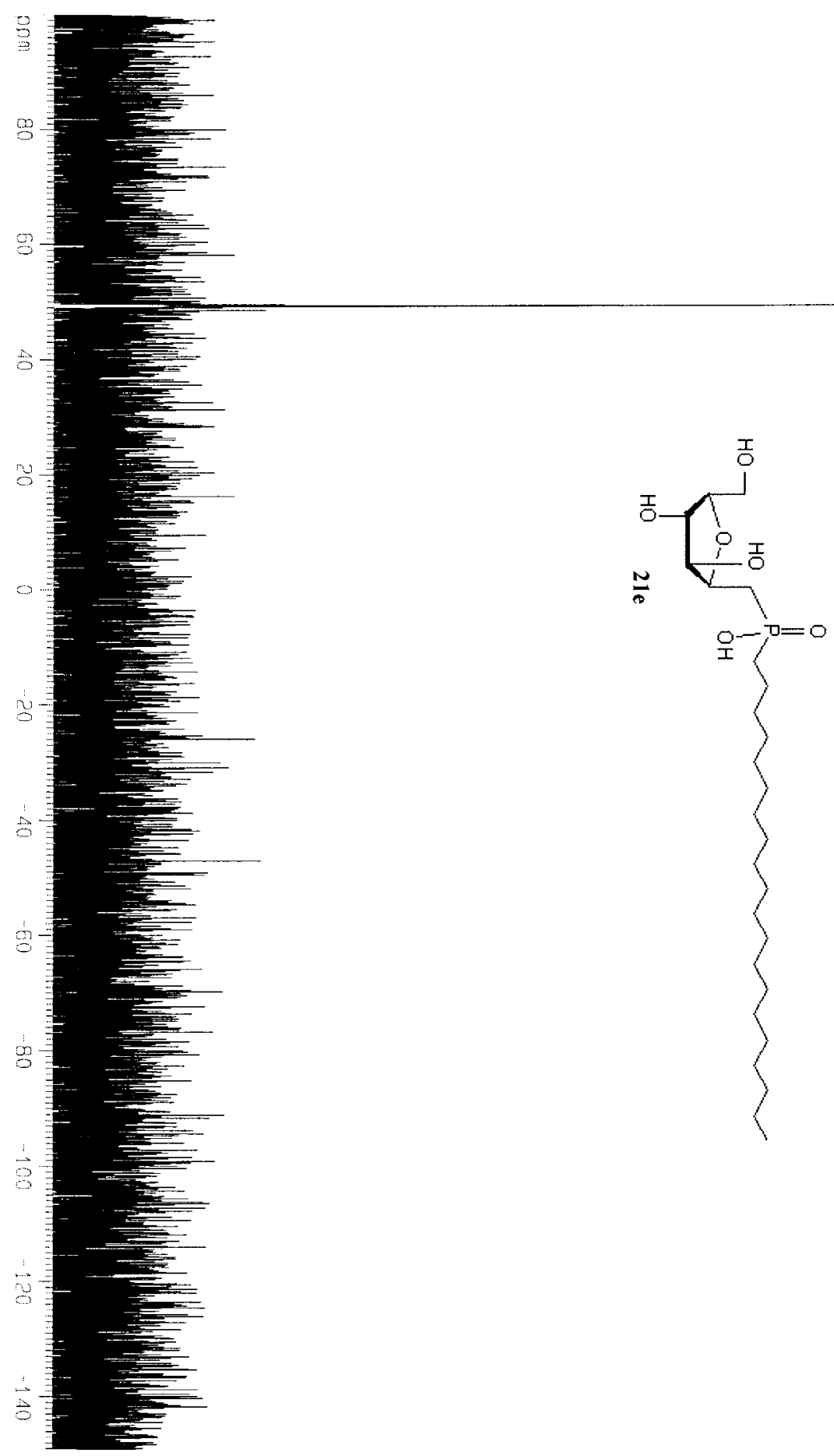

49.3060

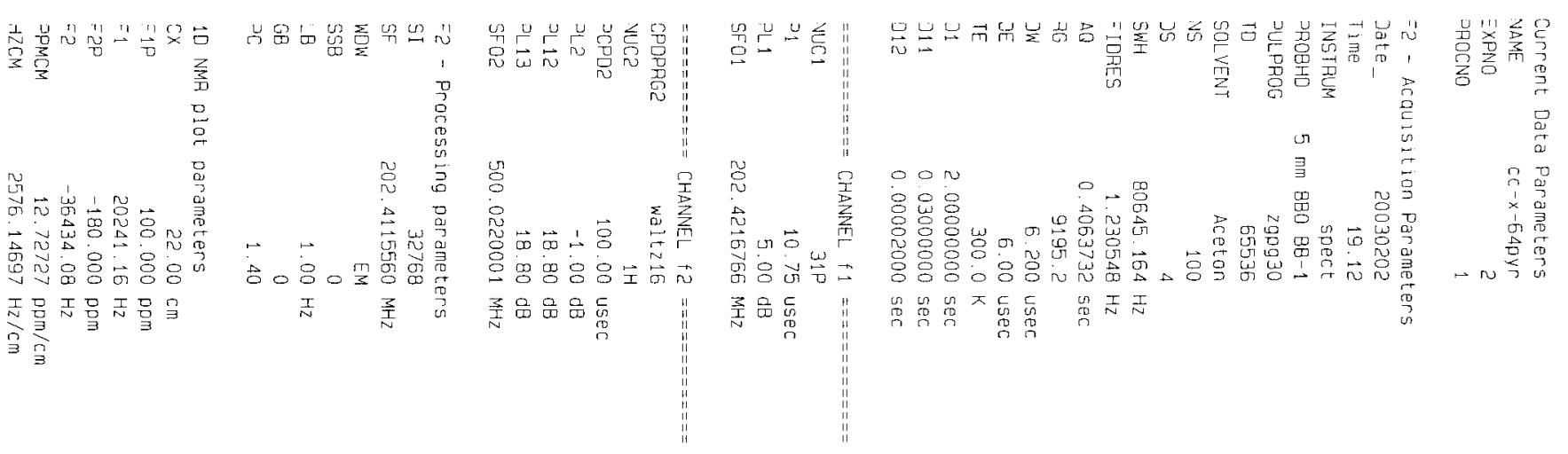



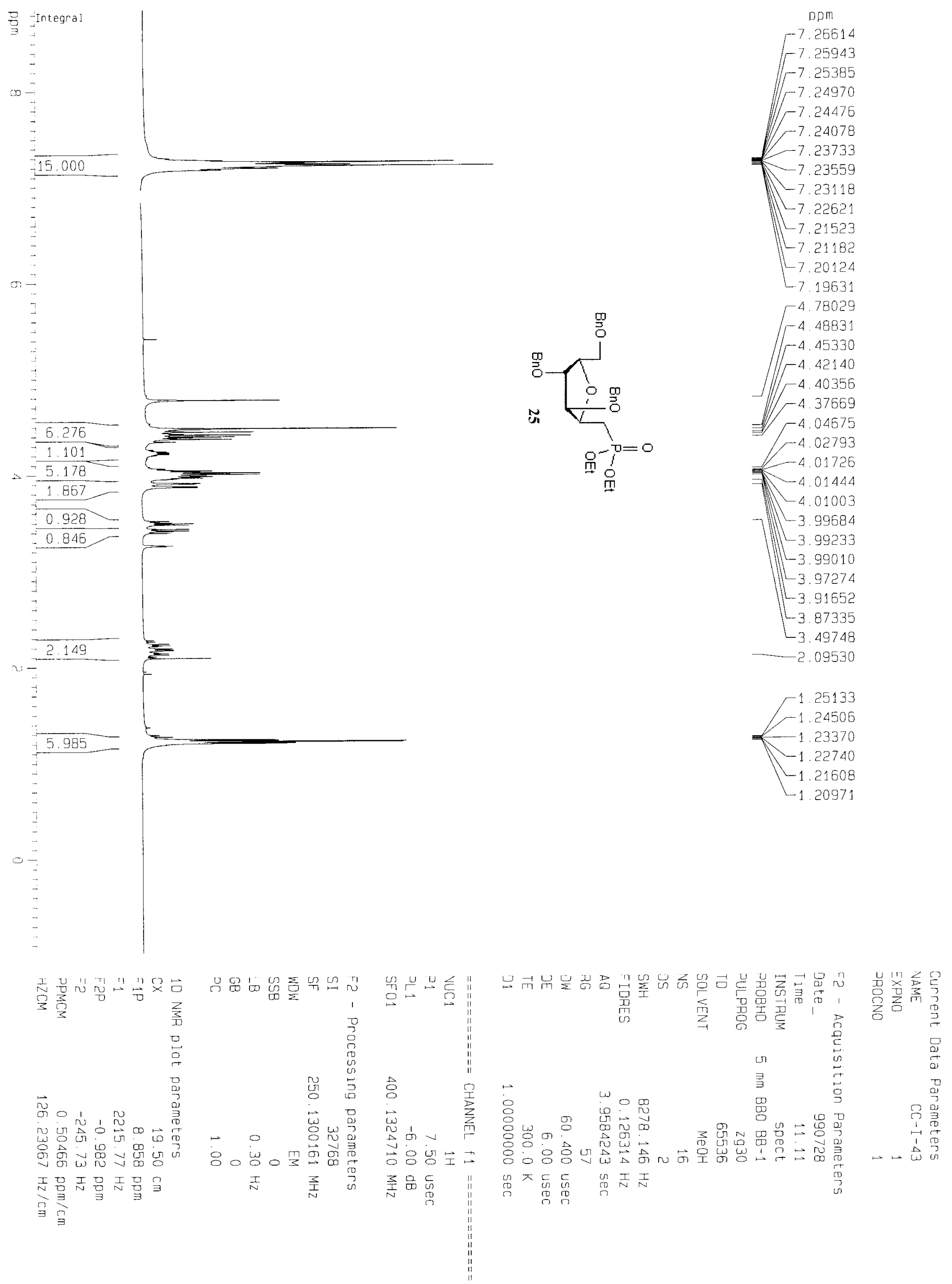

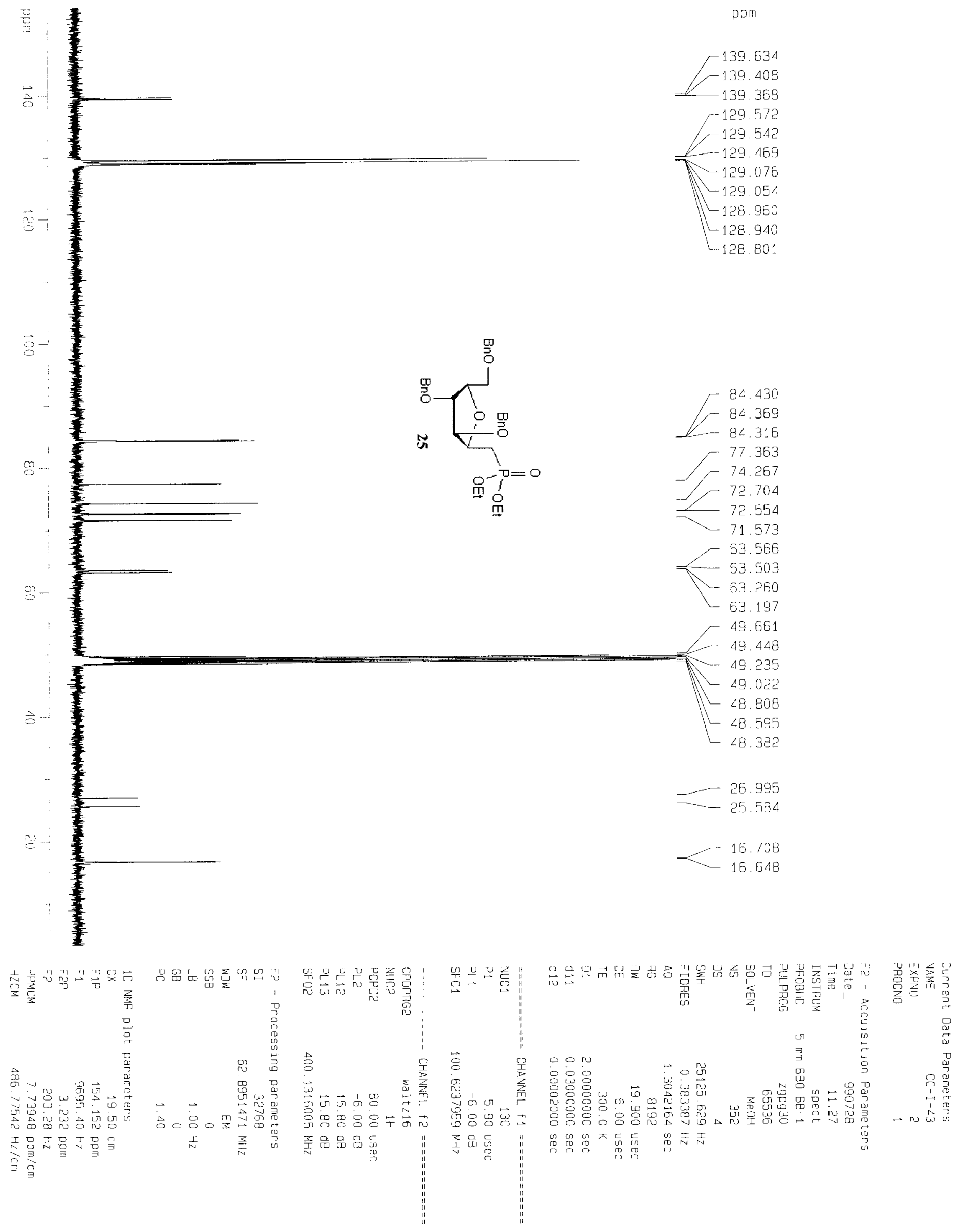


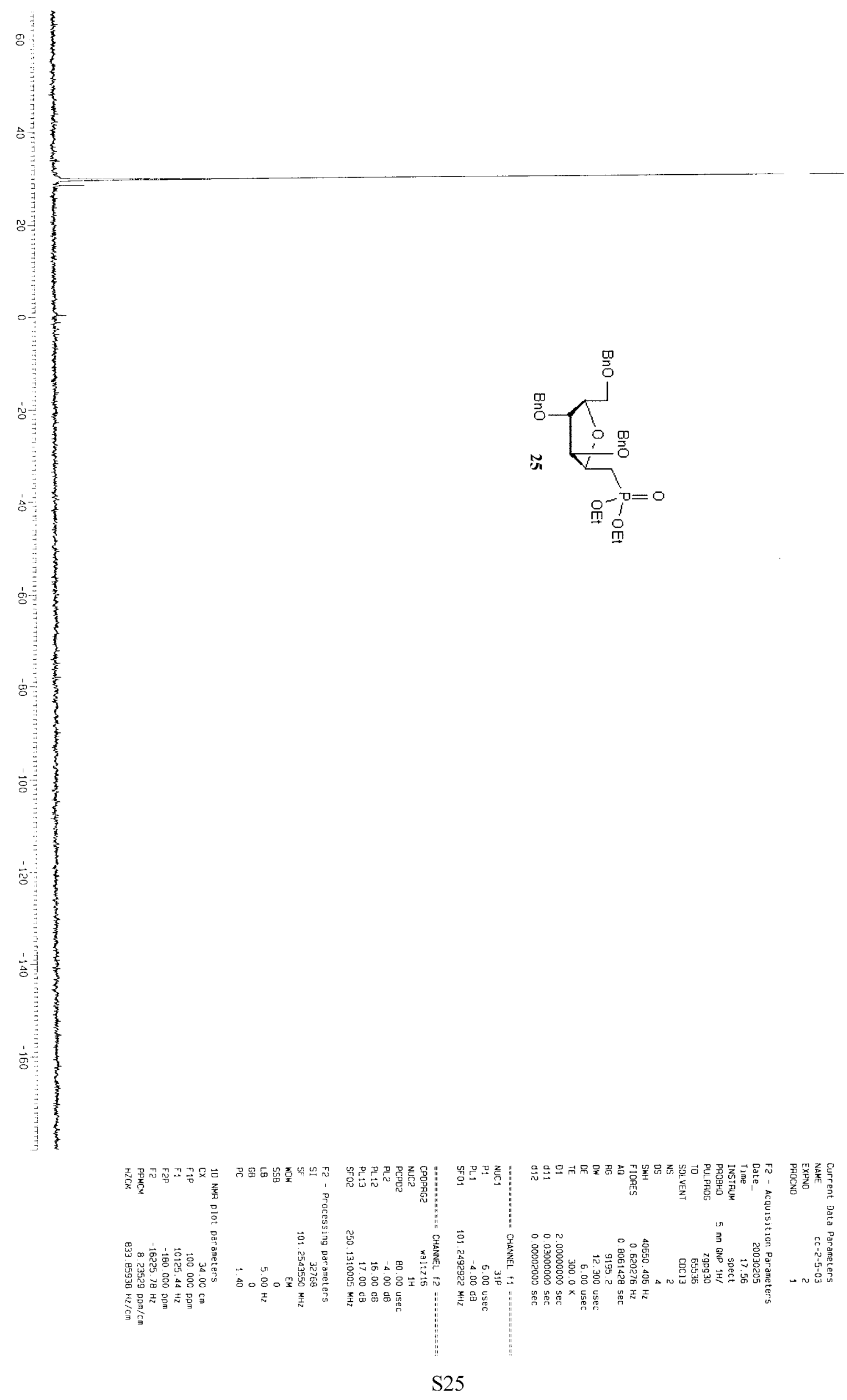

29.7790 


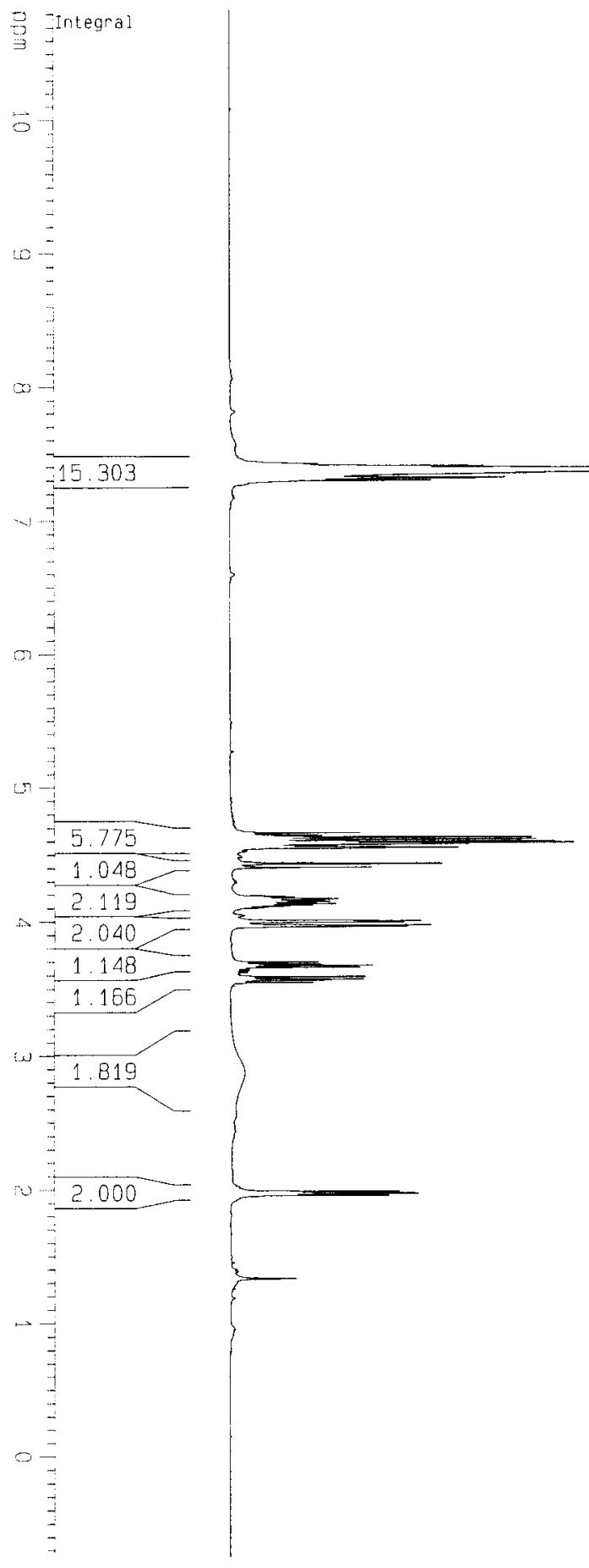

-7. 40892

$-7.40554$

$-7.39190$

$-7.37955$

$-7.36911$

$-7.36208$

$-7.35234$

$-7.34167$

$-7.33691$

$-7.32160$

$-7.31719$

-7.30185

4.66327

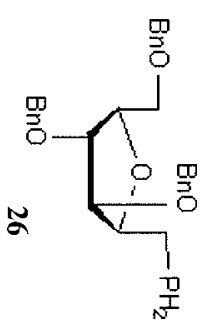

$-4.63306$

4.61658

$-4.59856$

II -4.58810

$-4.58241$

$-4.55779$

55241

$-4.43647$

$-4.40710$

$-4.00392$

$7\lfloor-3.99720$

$-3.97142$

$-3.96278$

$-3.67369$

$-3.65913$

$-3.58912$

L-3.57223

7 -3.56462

$-1.98652$

$-1.97499$

$L_{1} .96868$

$L_{1} .95720$

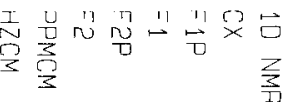

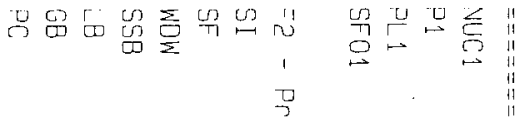

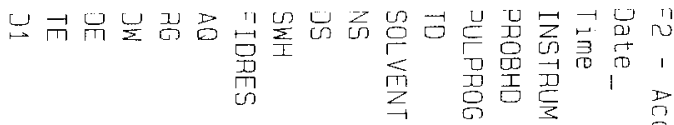

号紧希交的

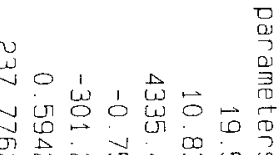

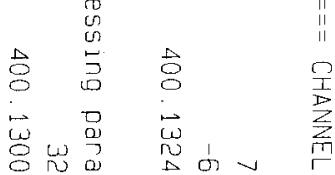

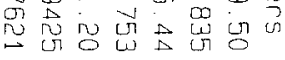

志号甹枵甹号号

8

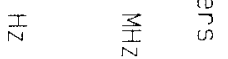

蛋吕岳

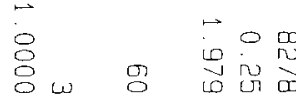

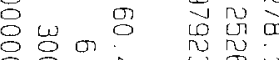

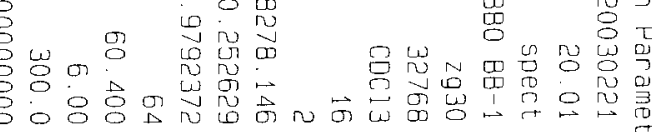

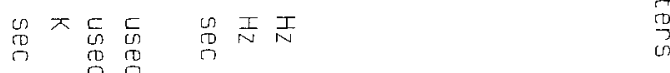

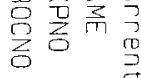

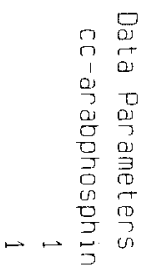

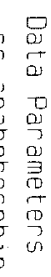



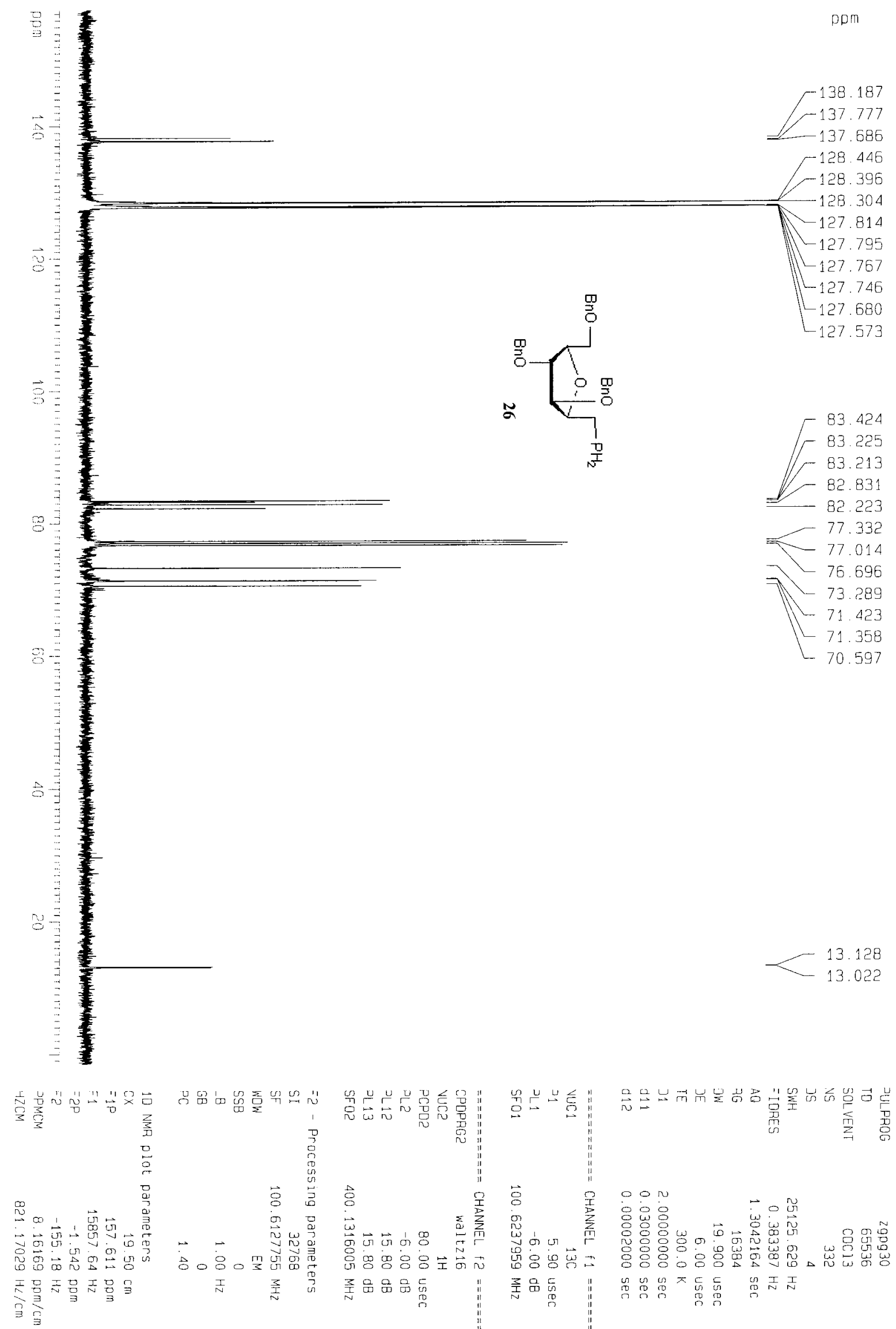


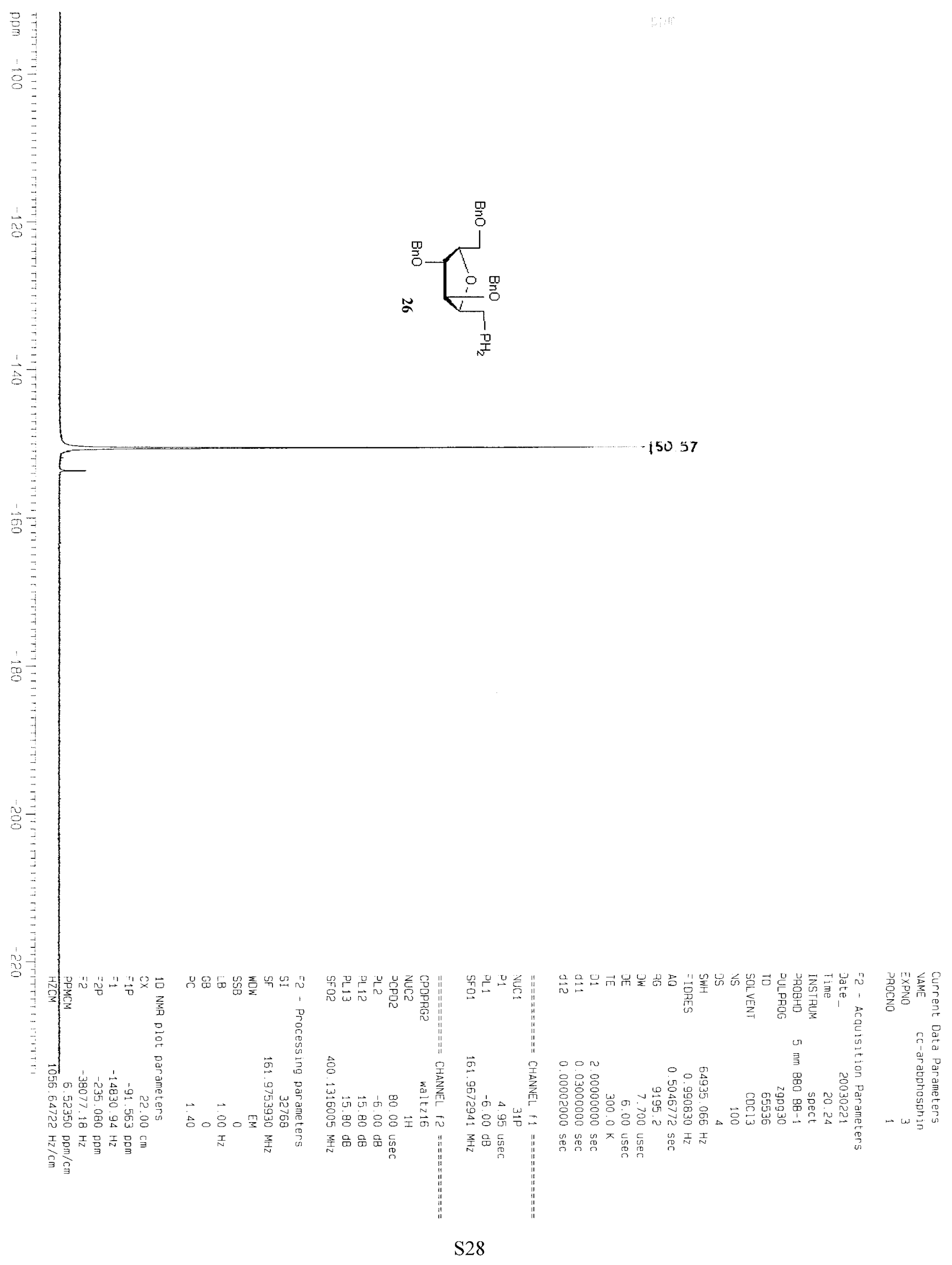




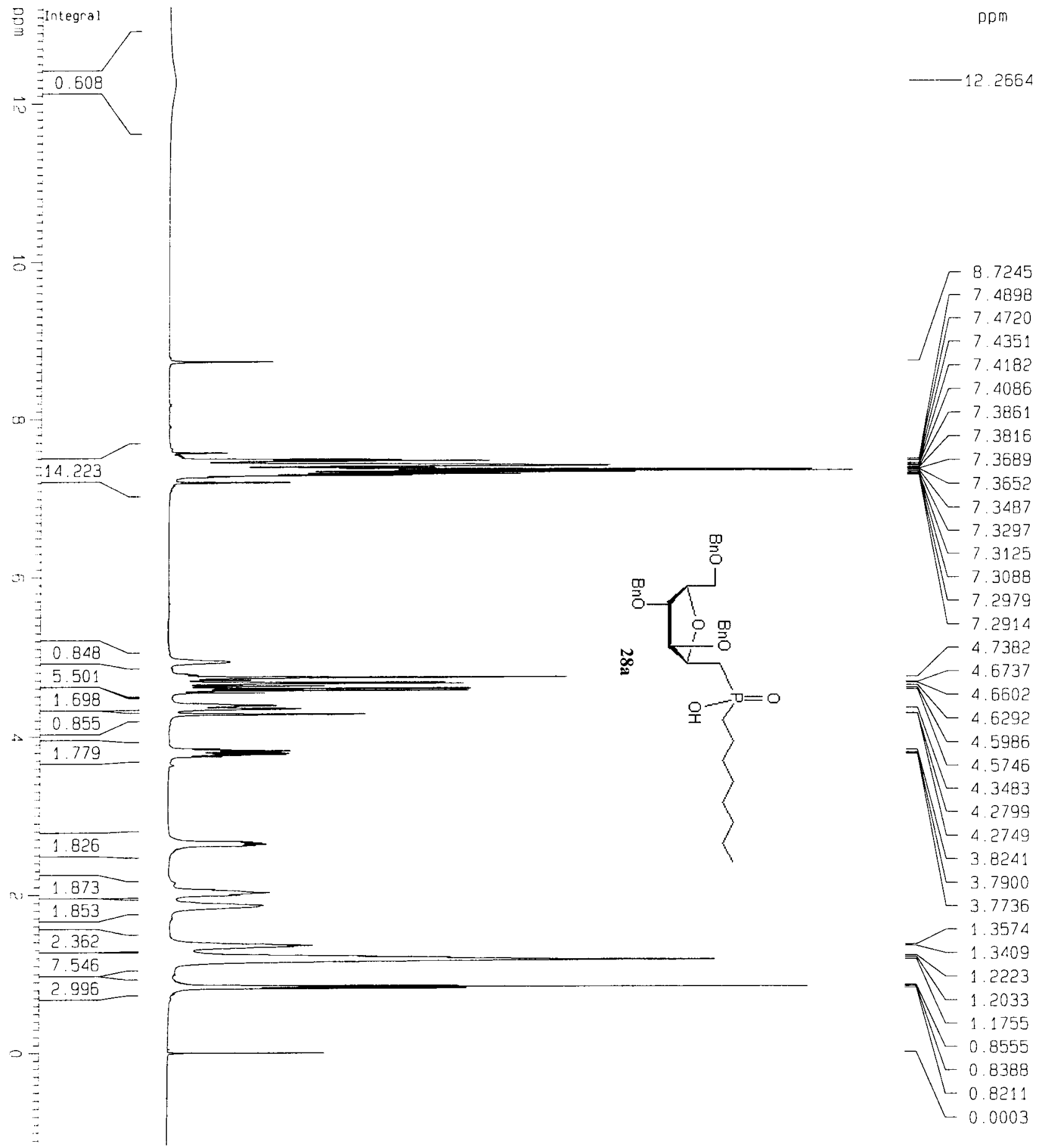

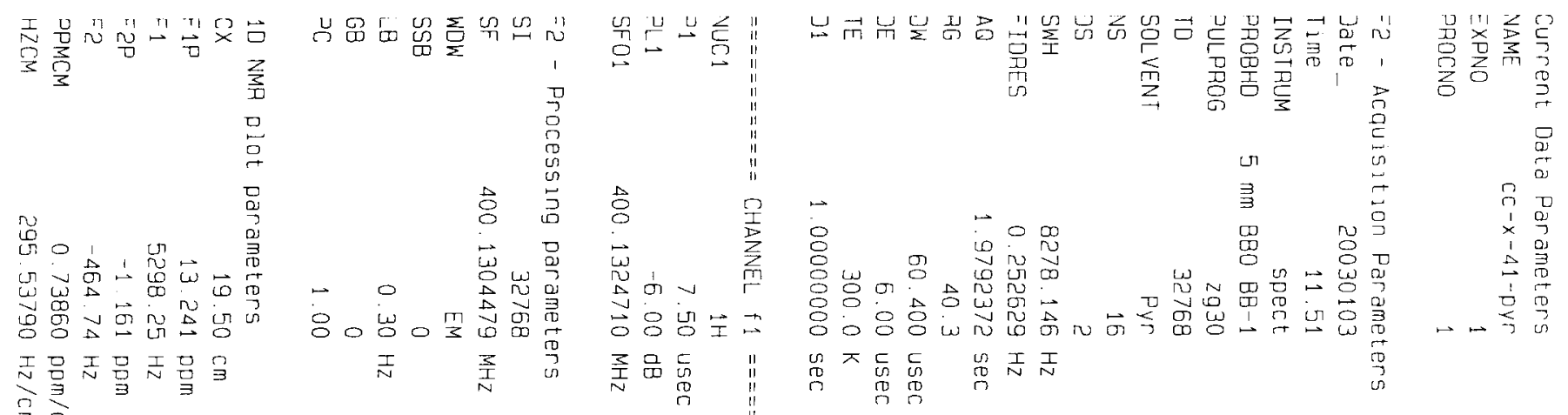




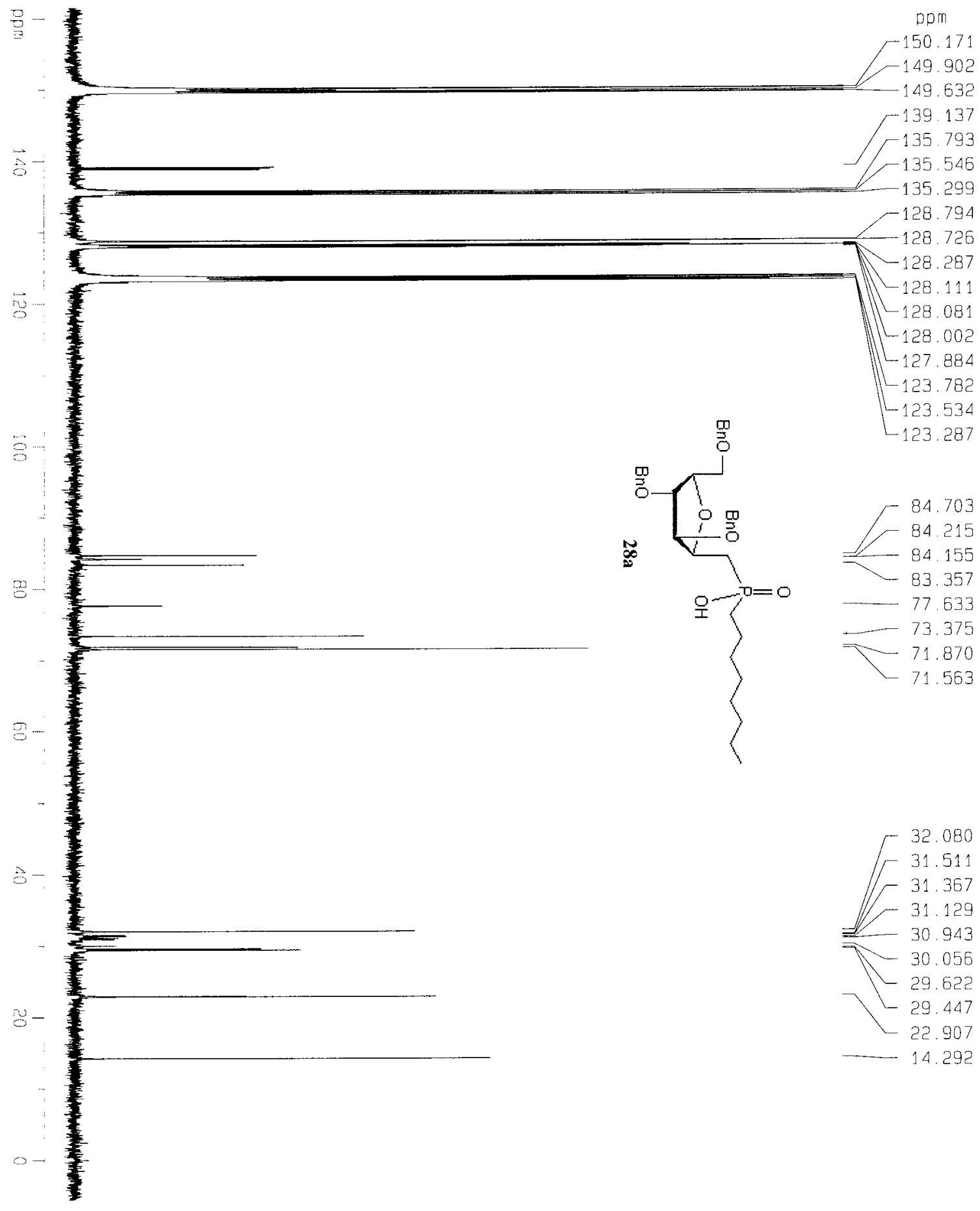

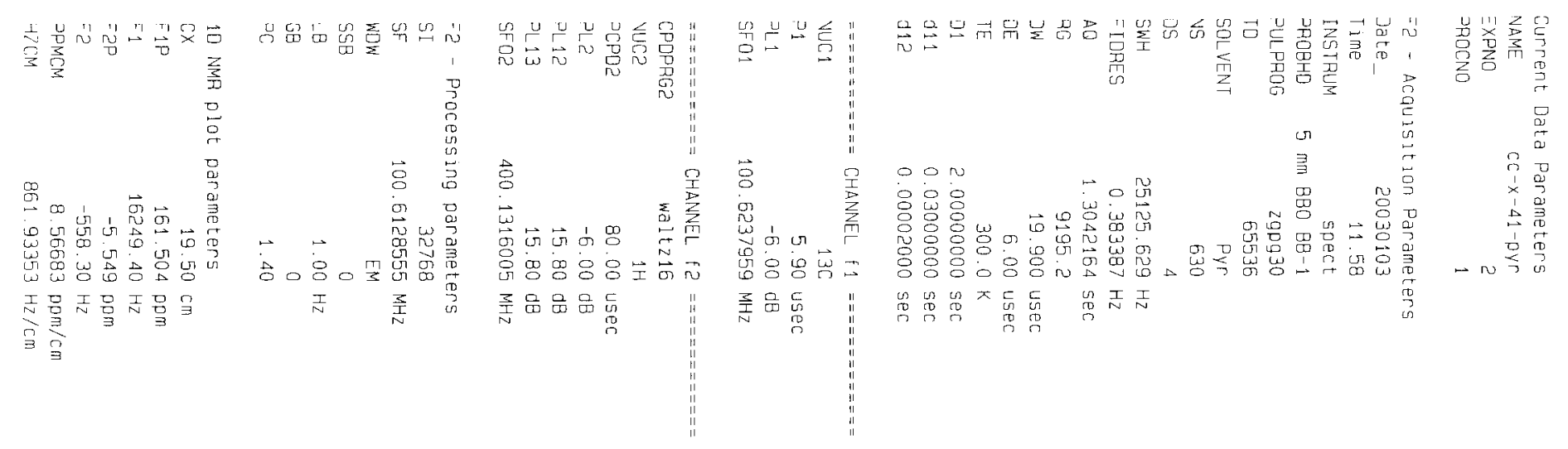




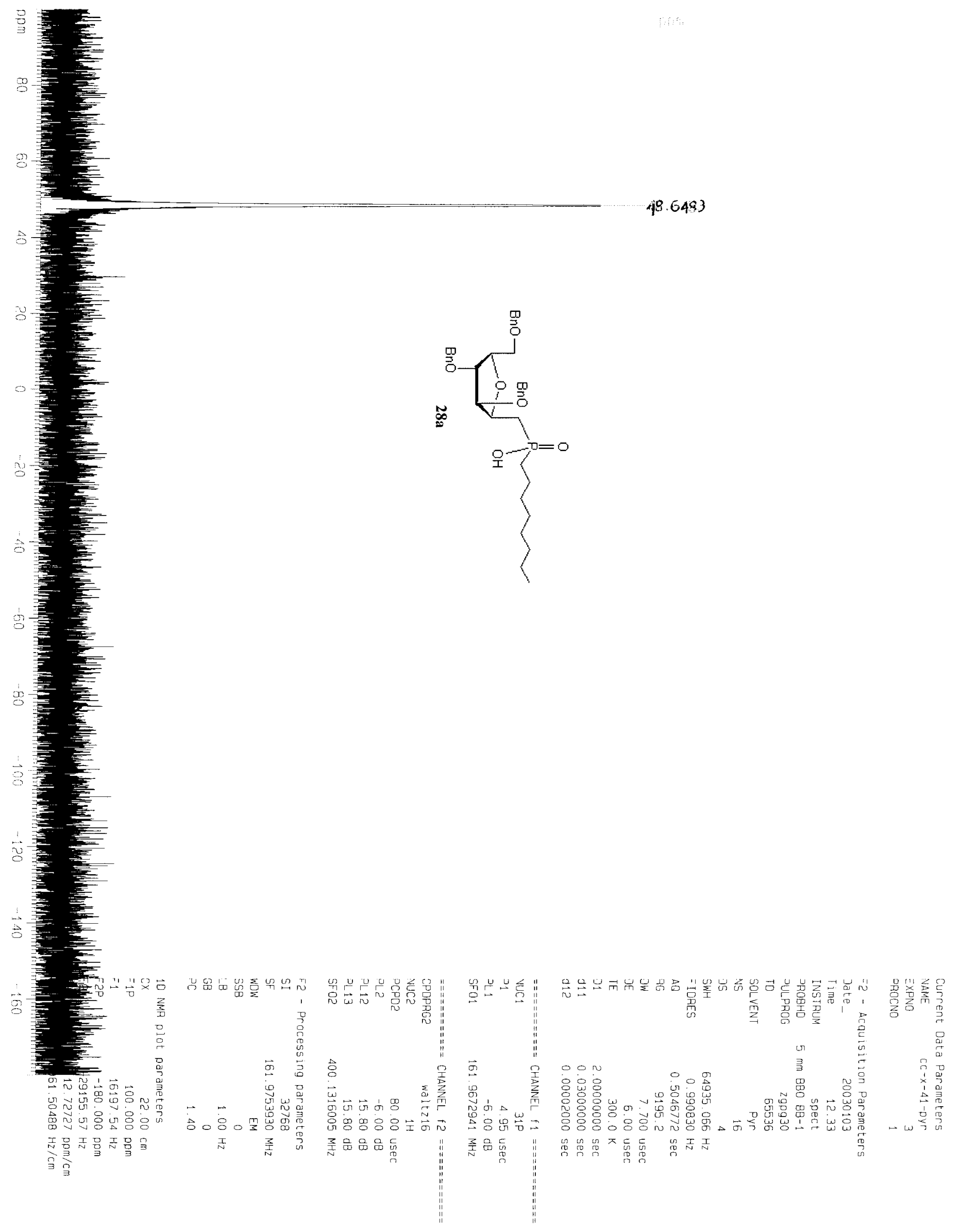




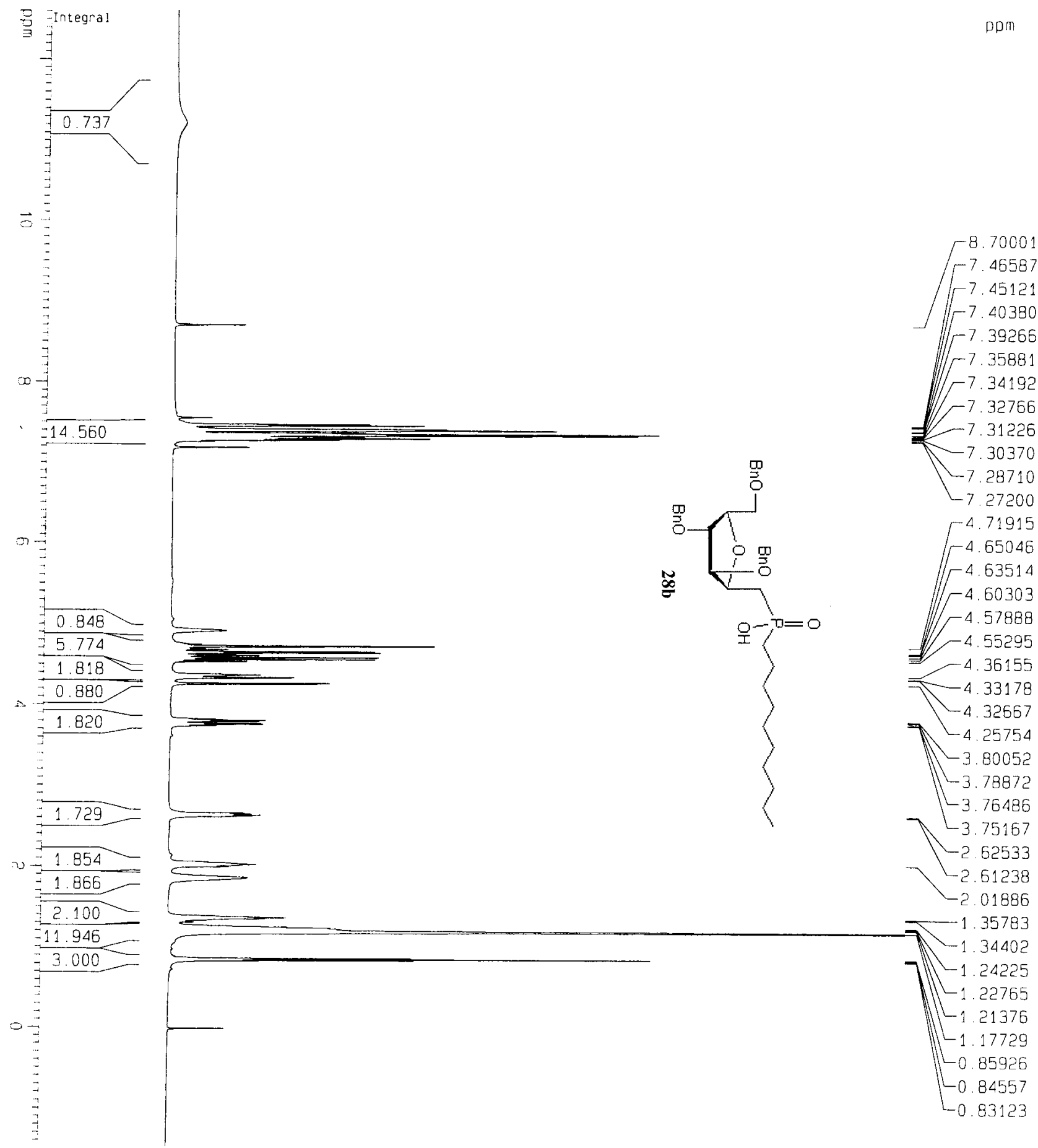

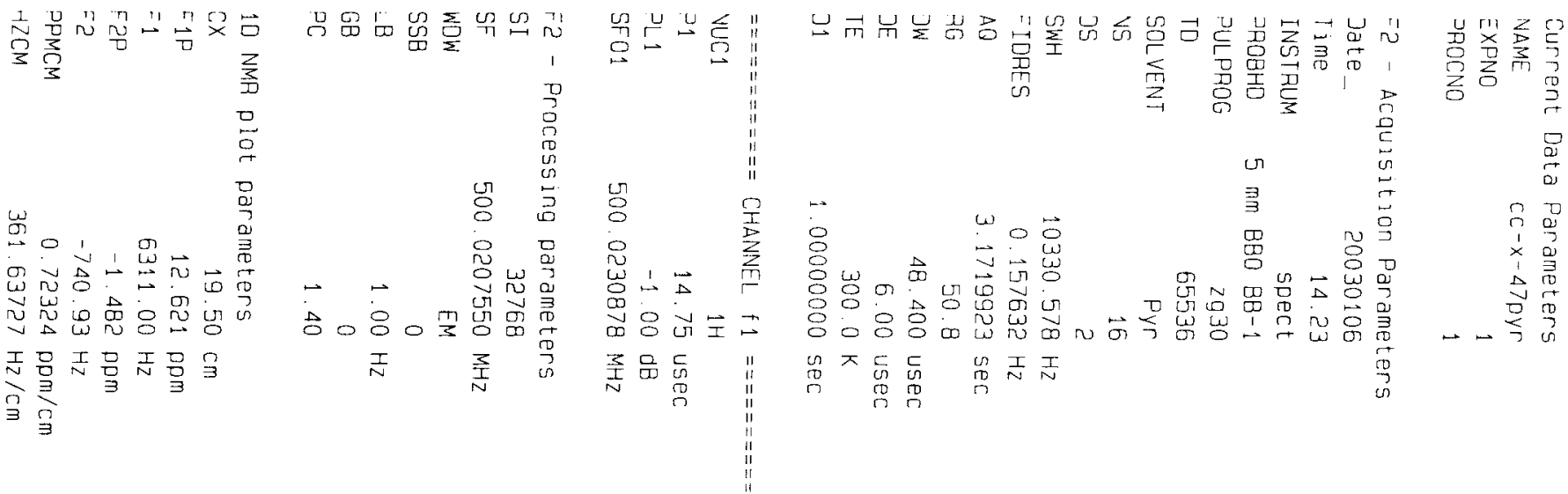




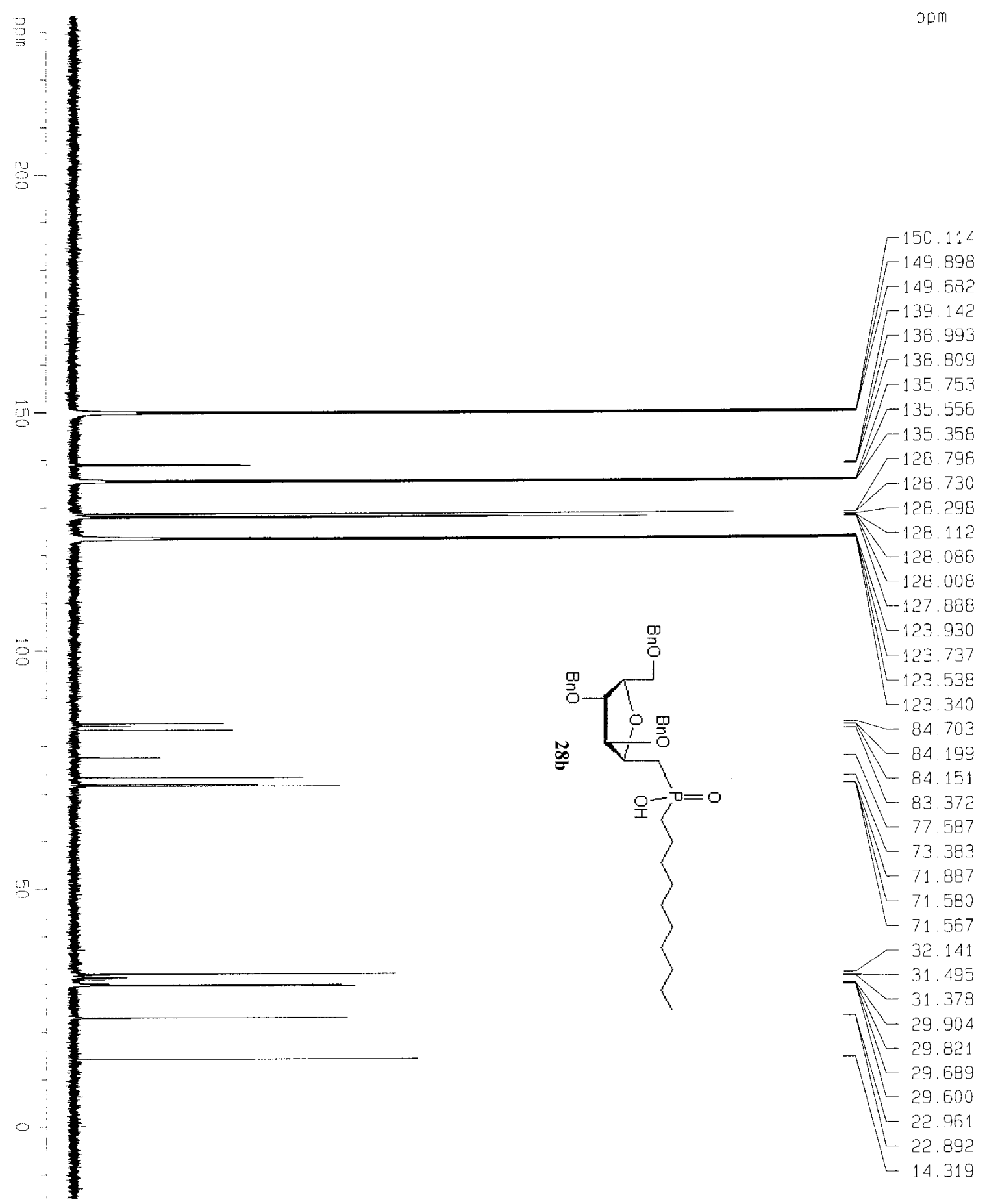

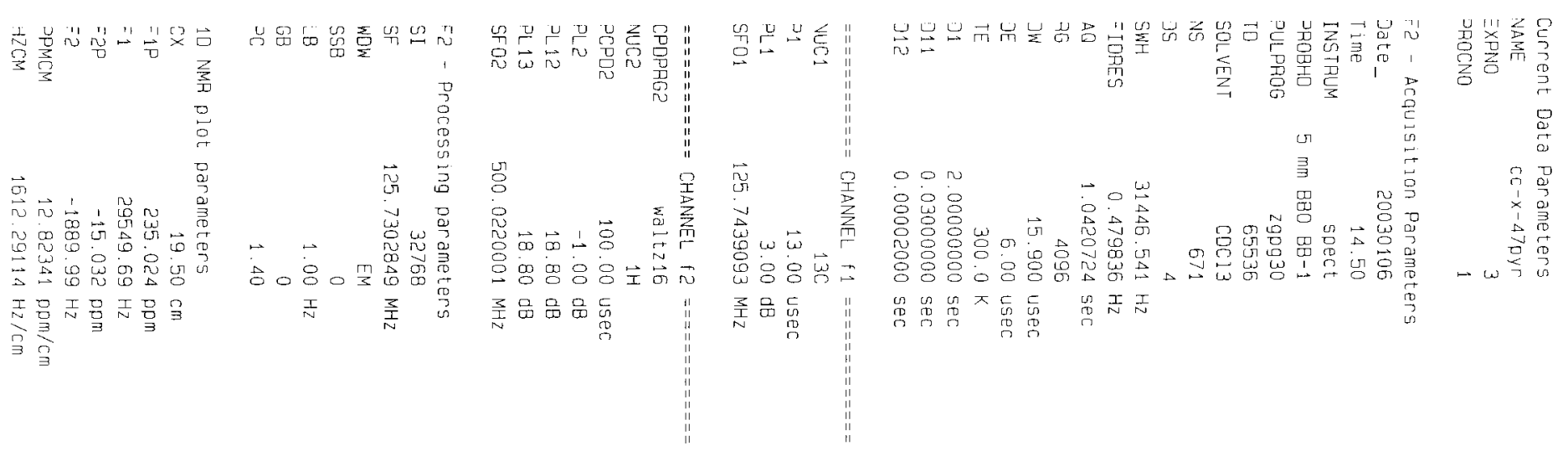




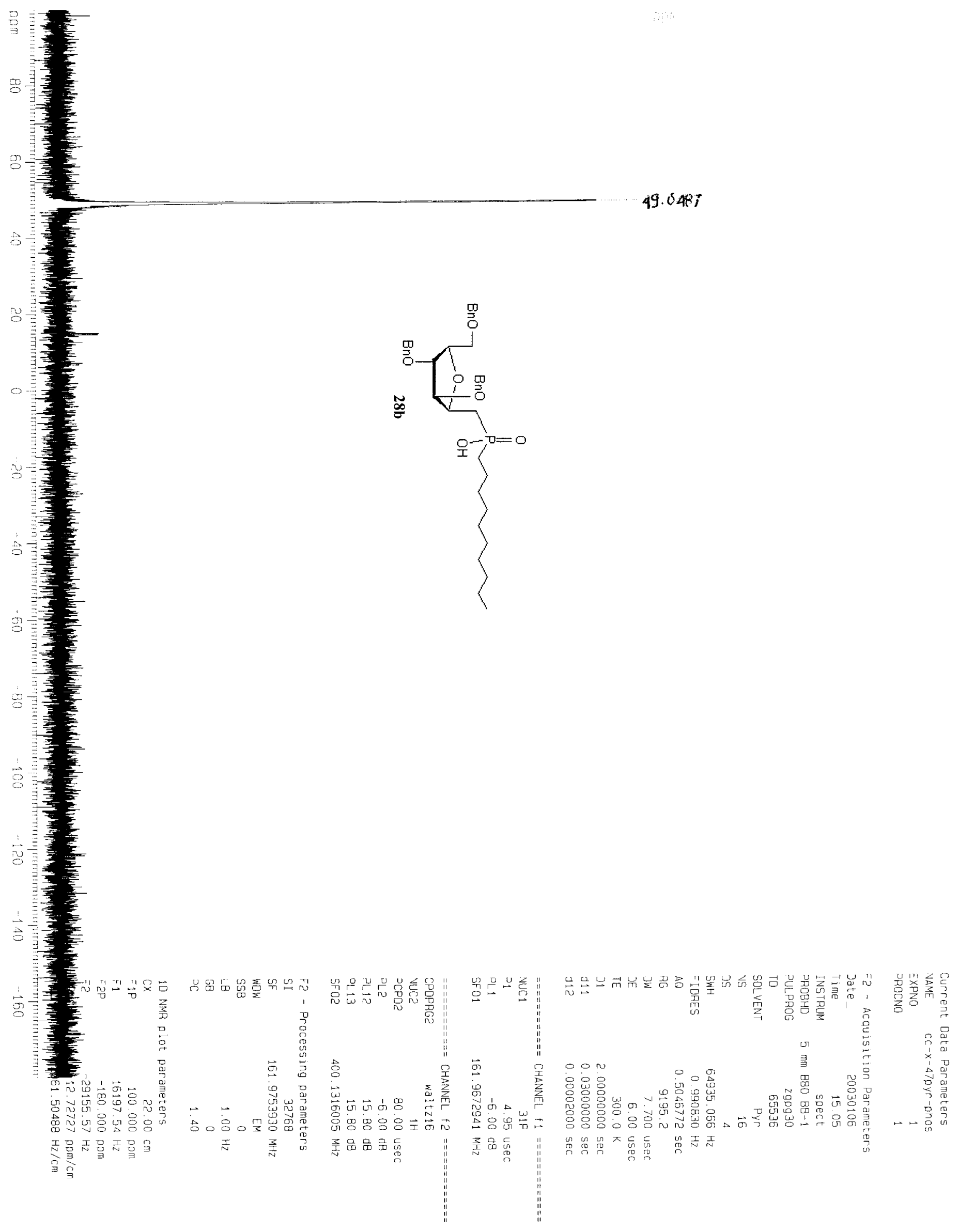




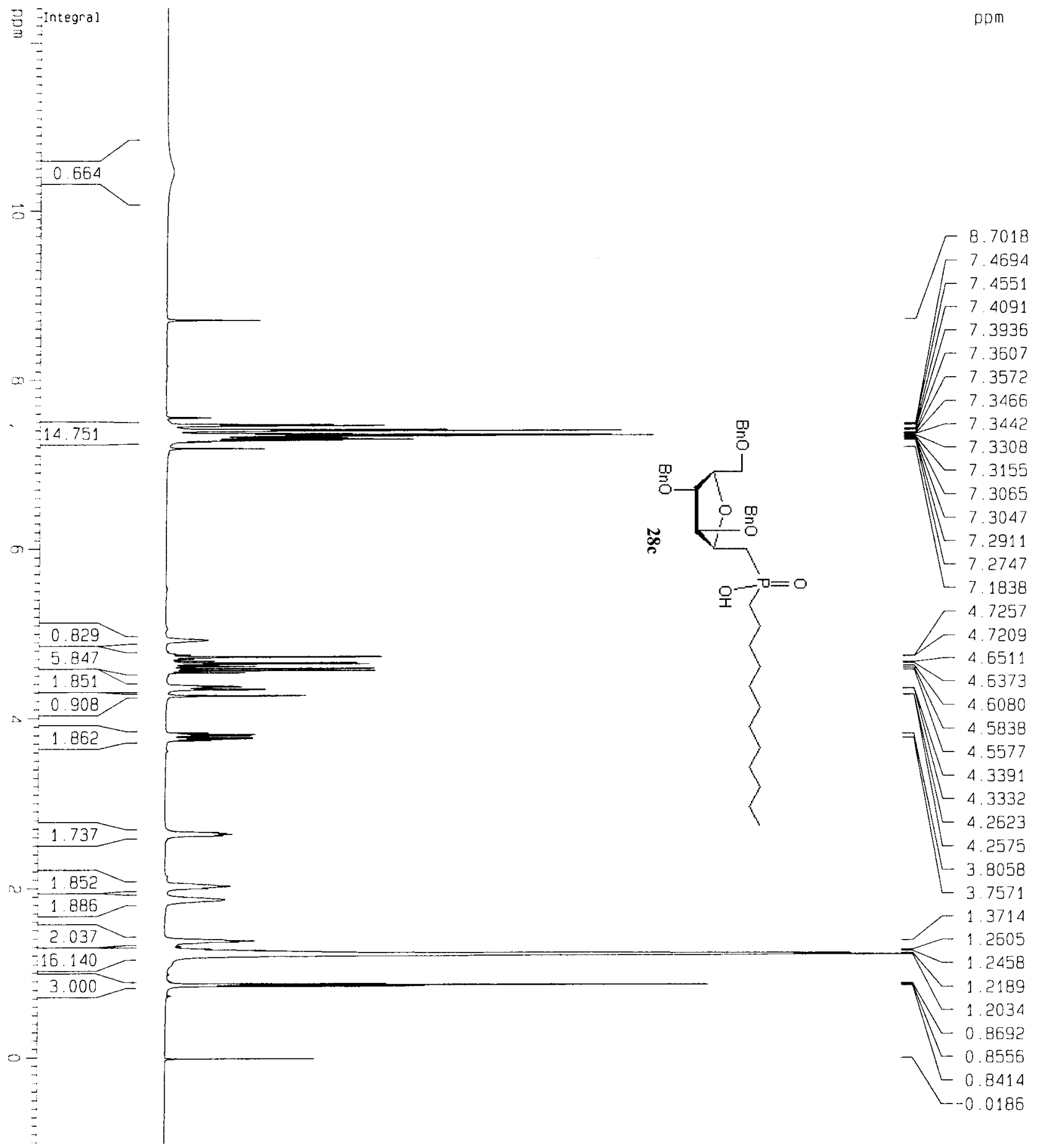

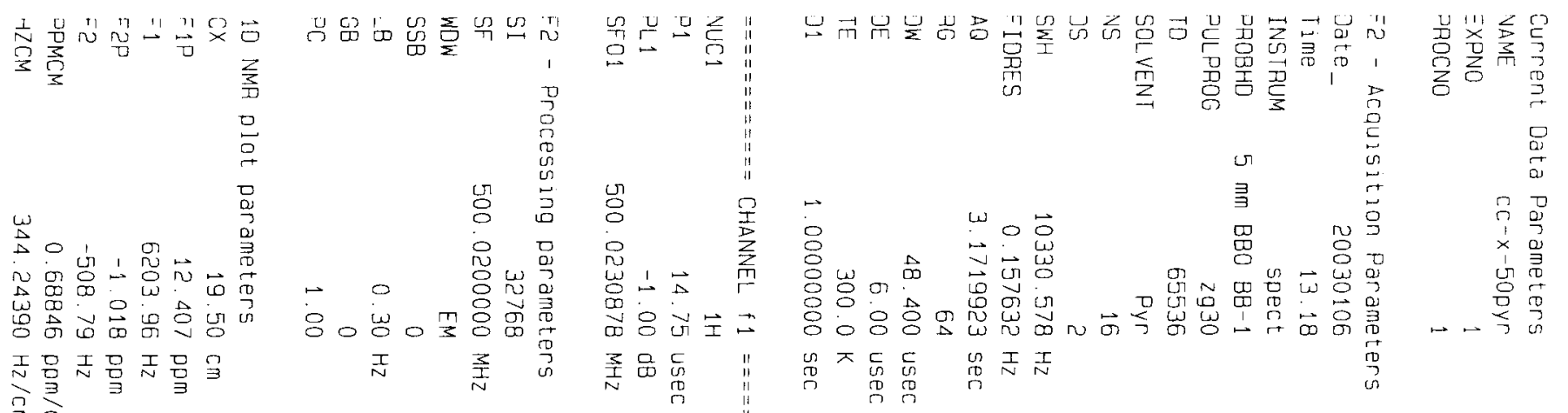



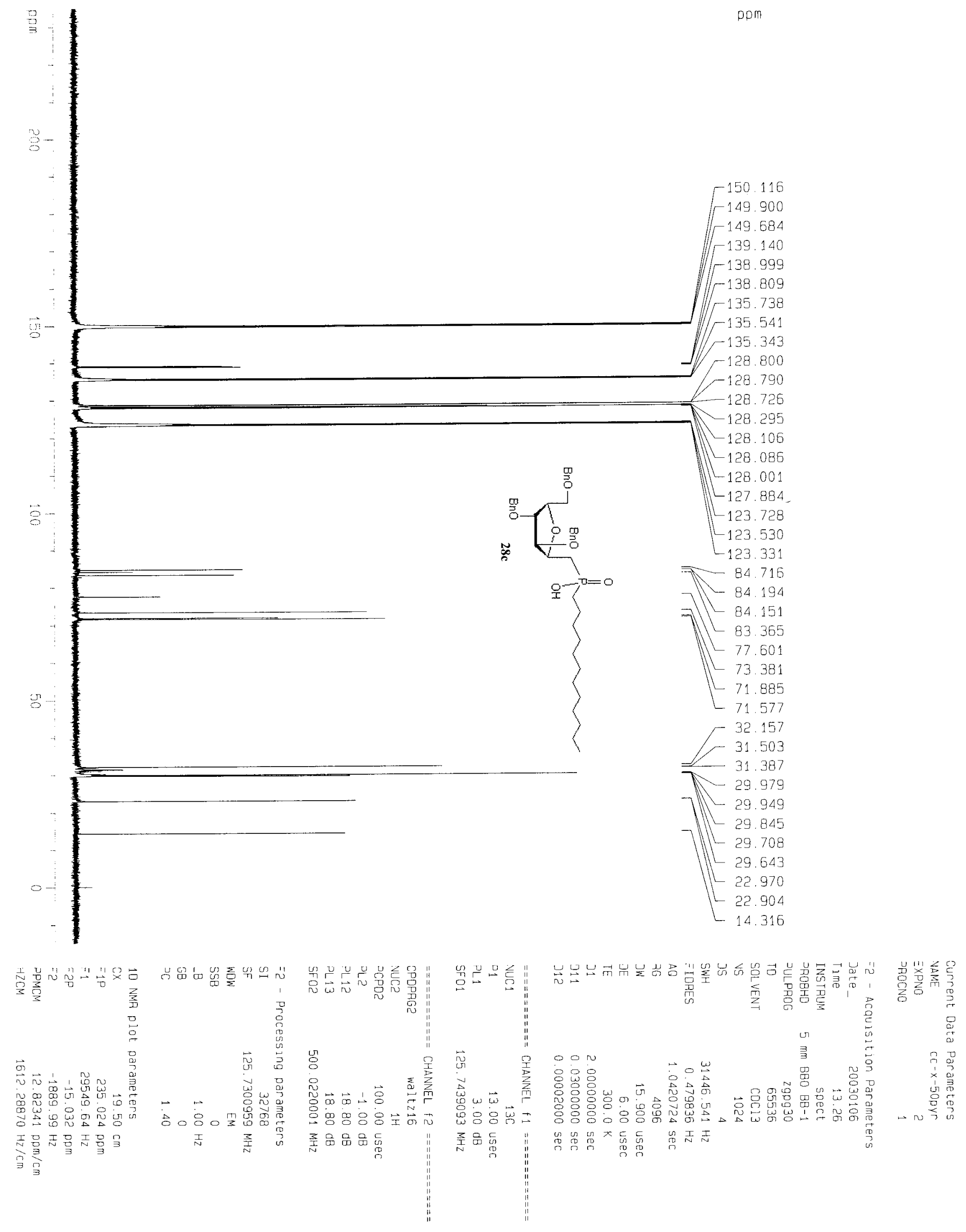


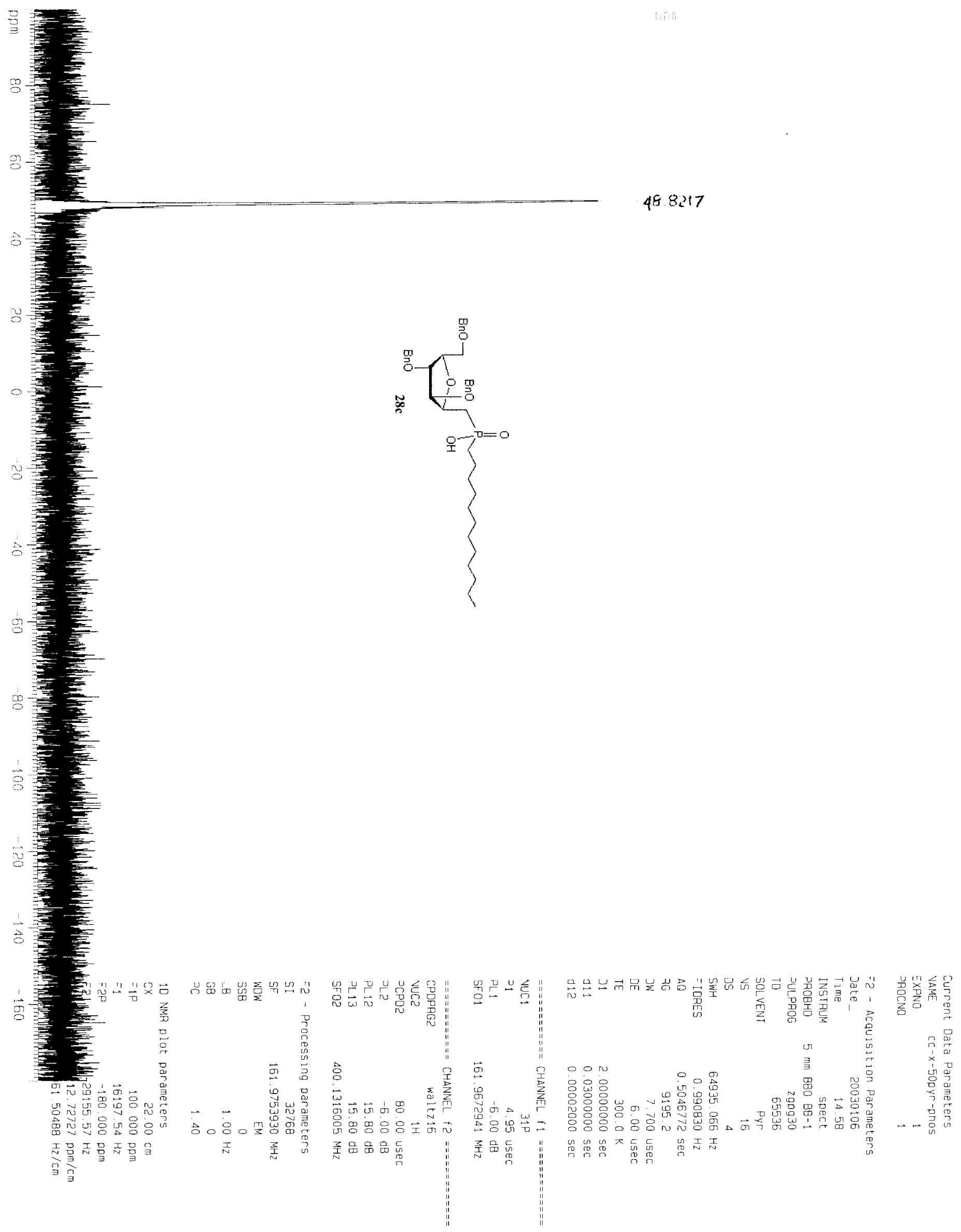




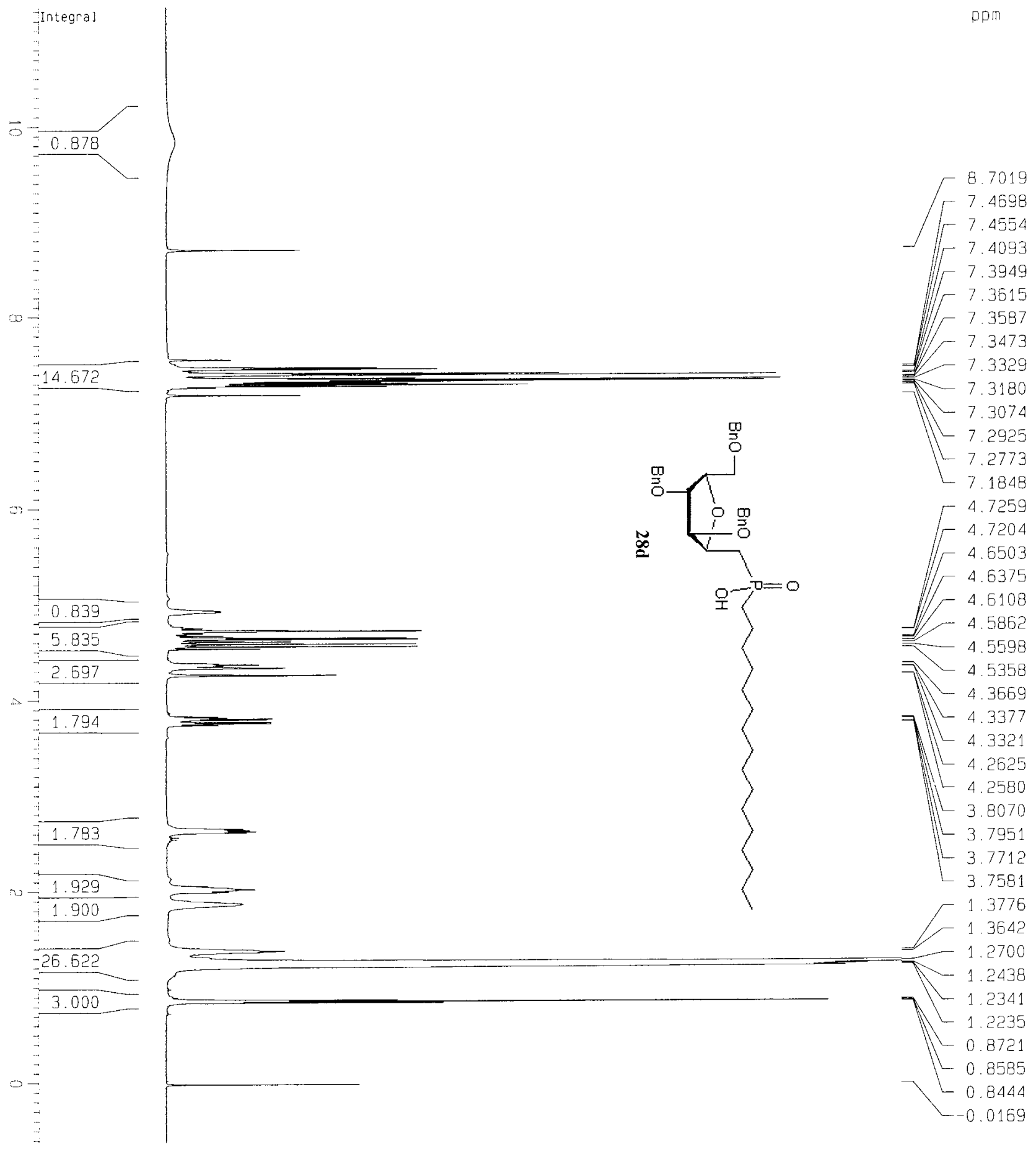

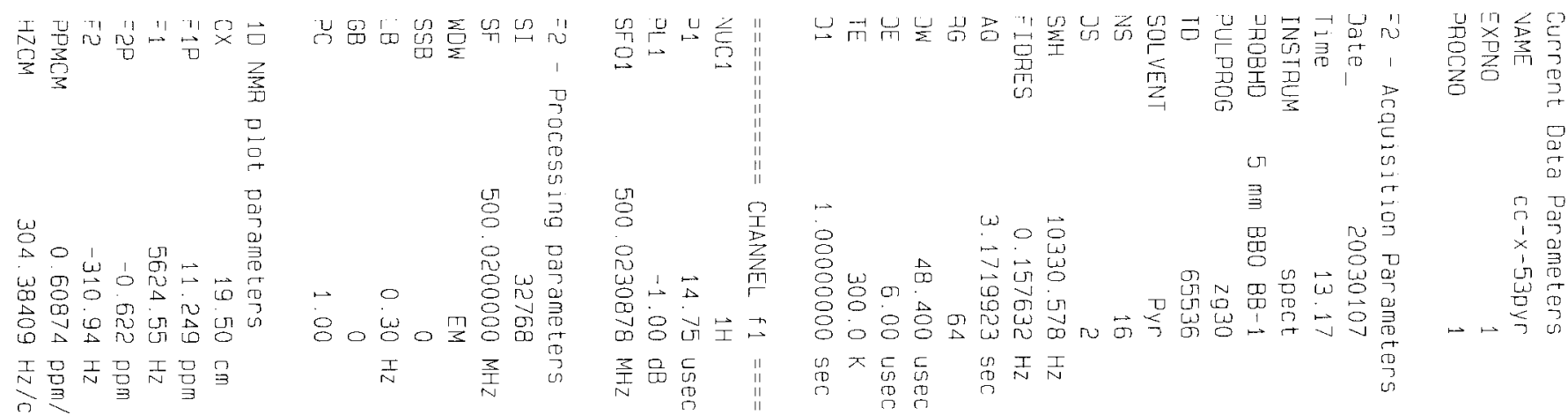




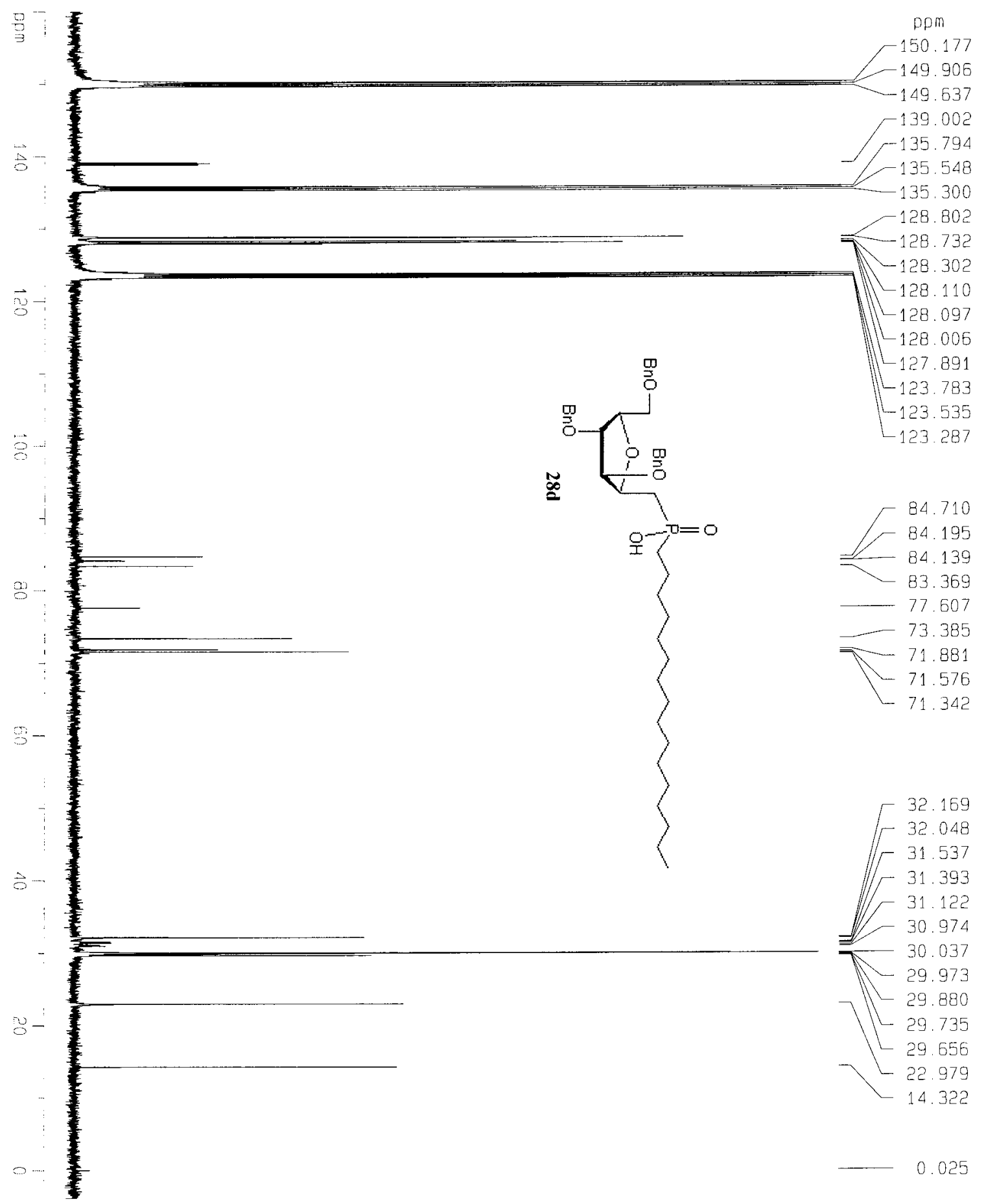

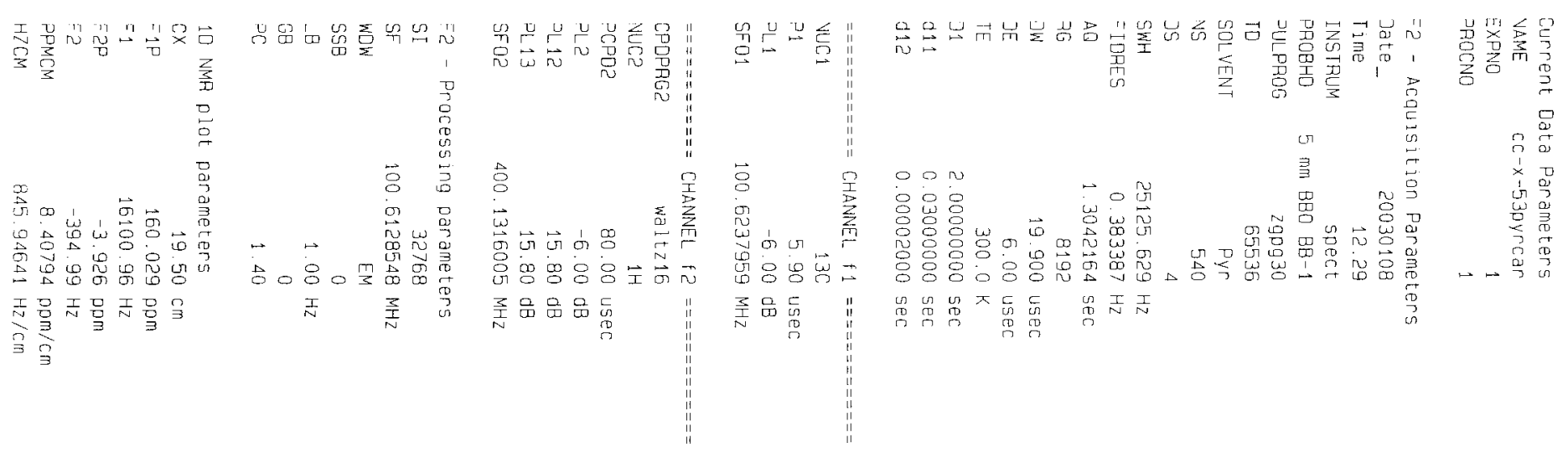




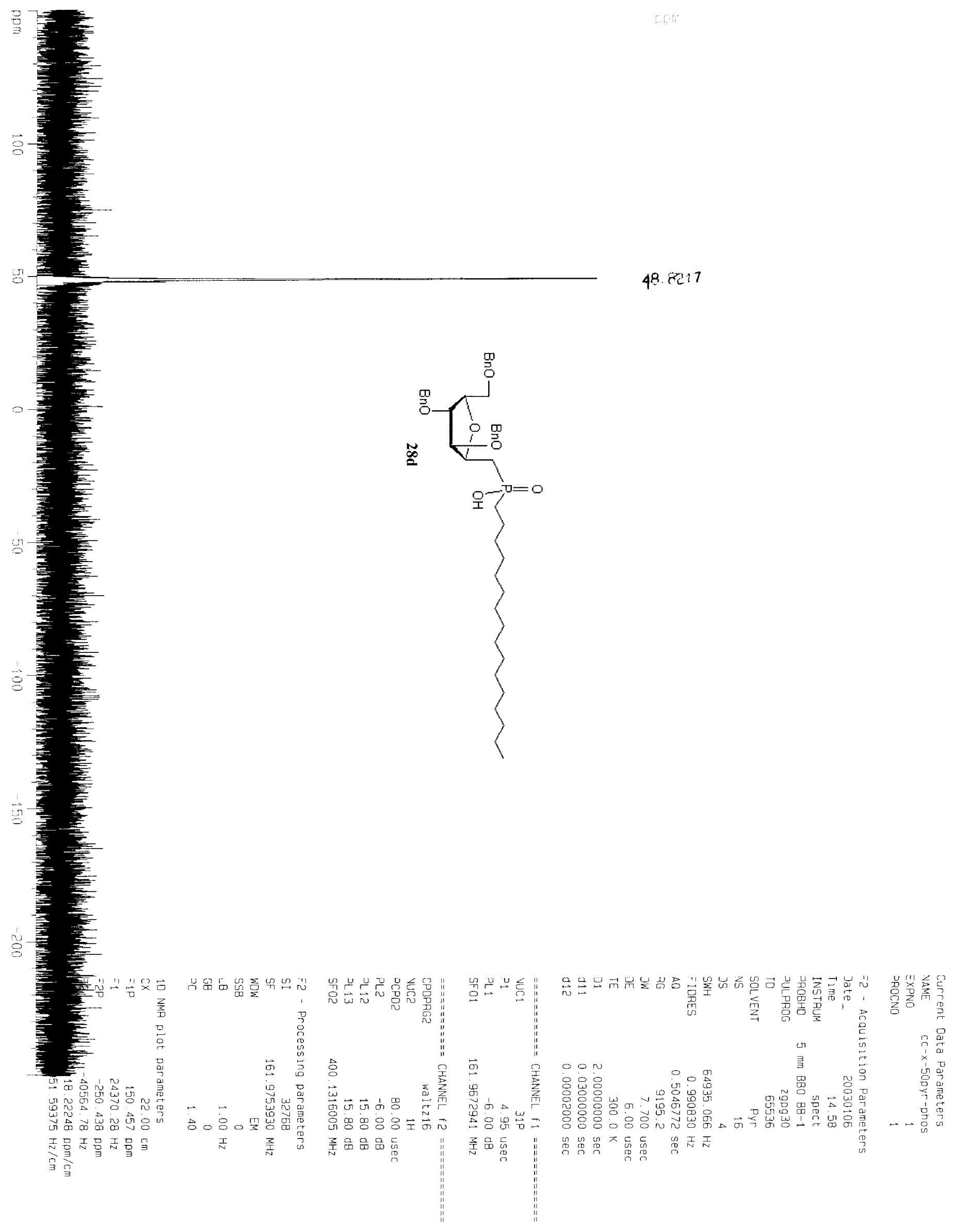




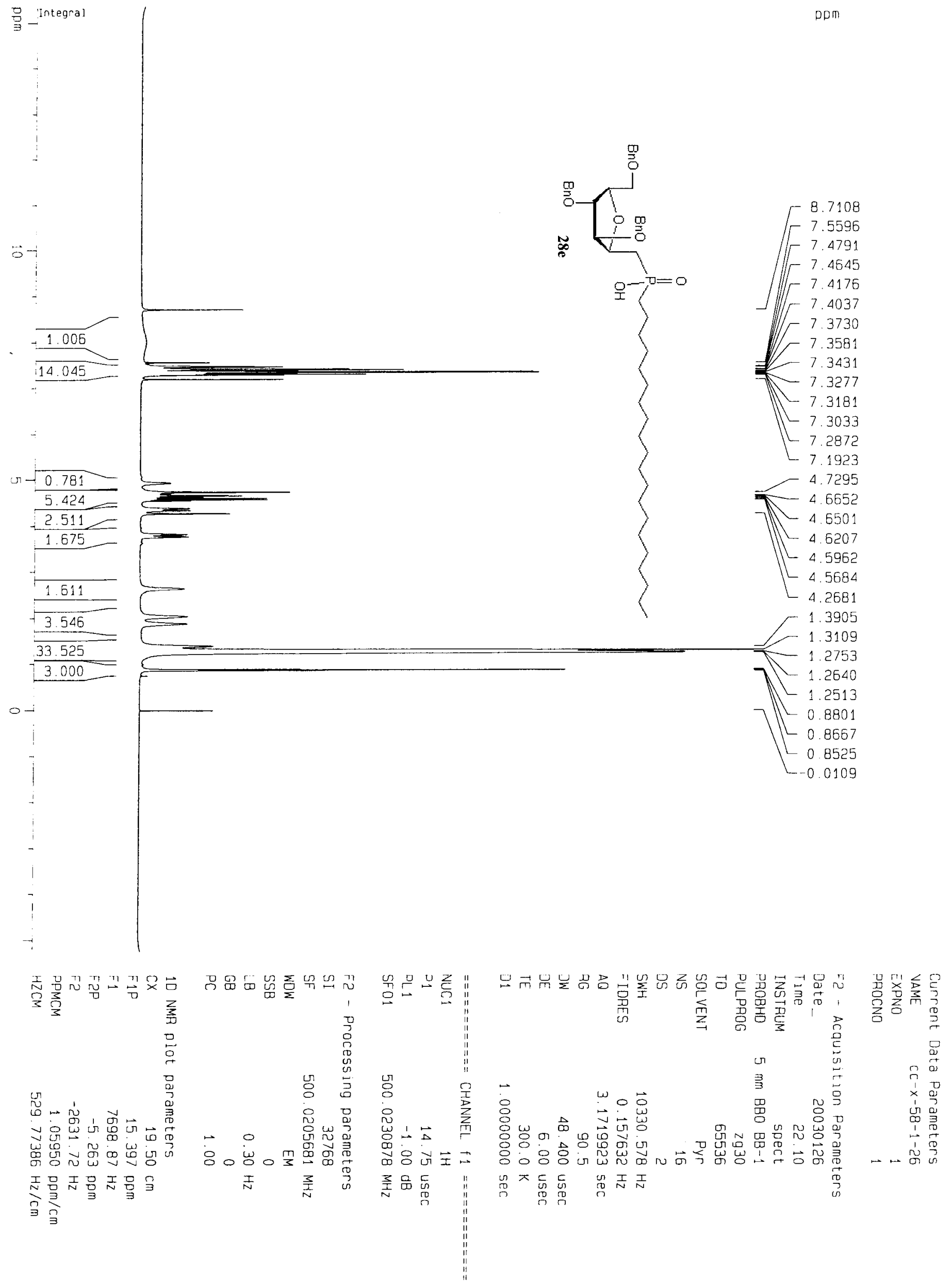




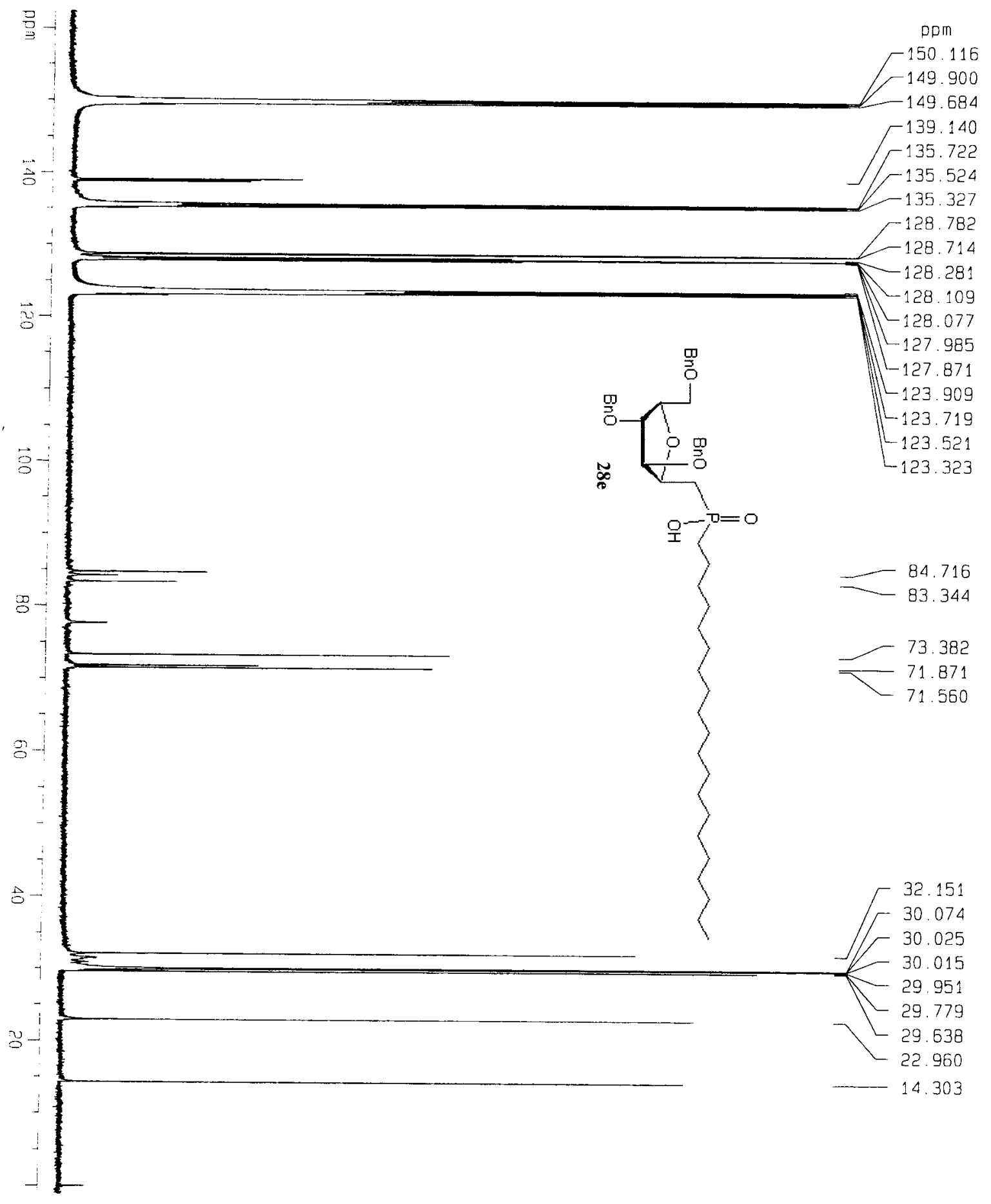

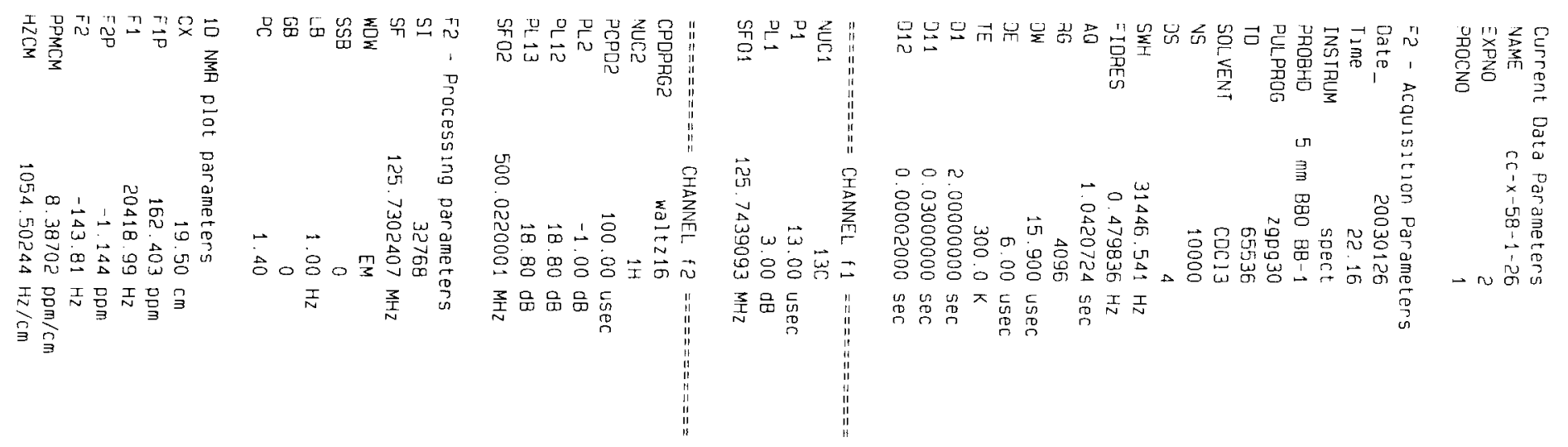




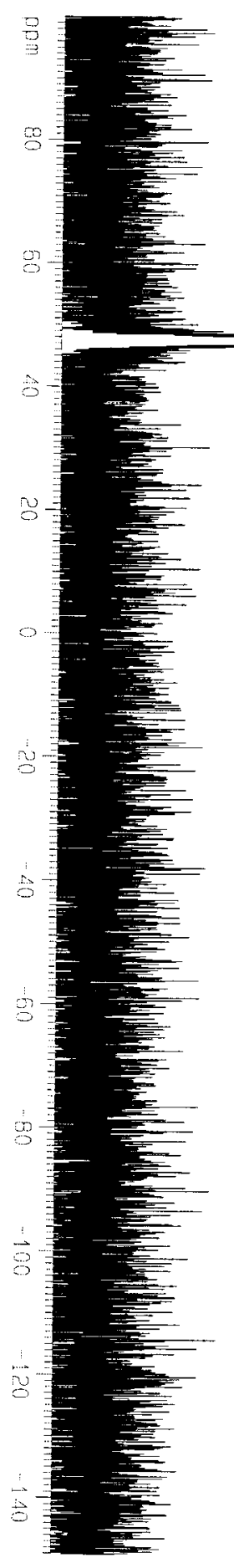

47395

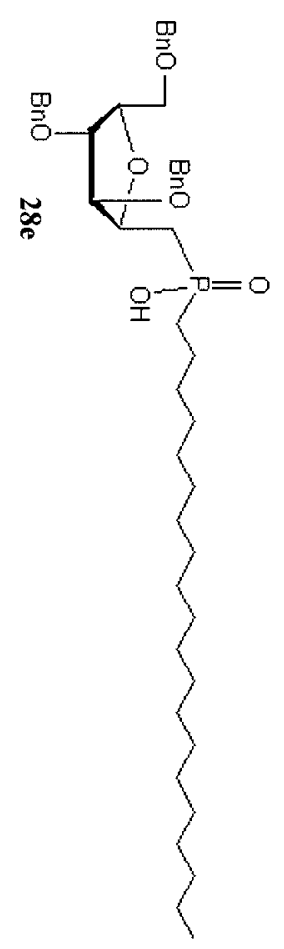

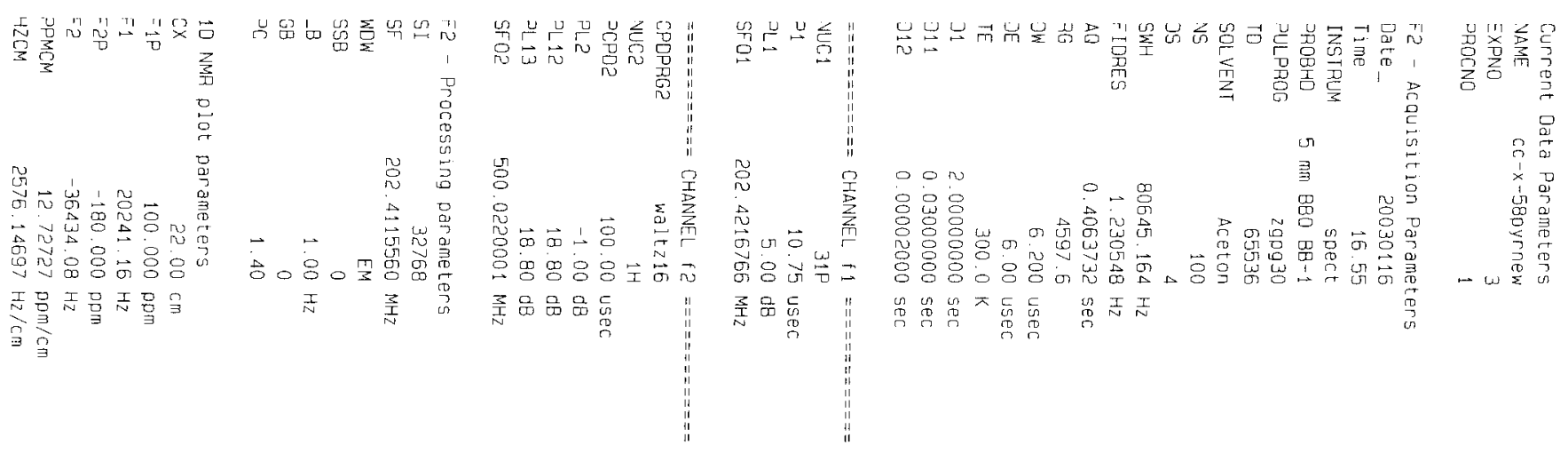




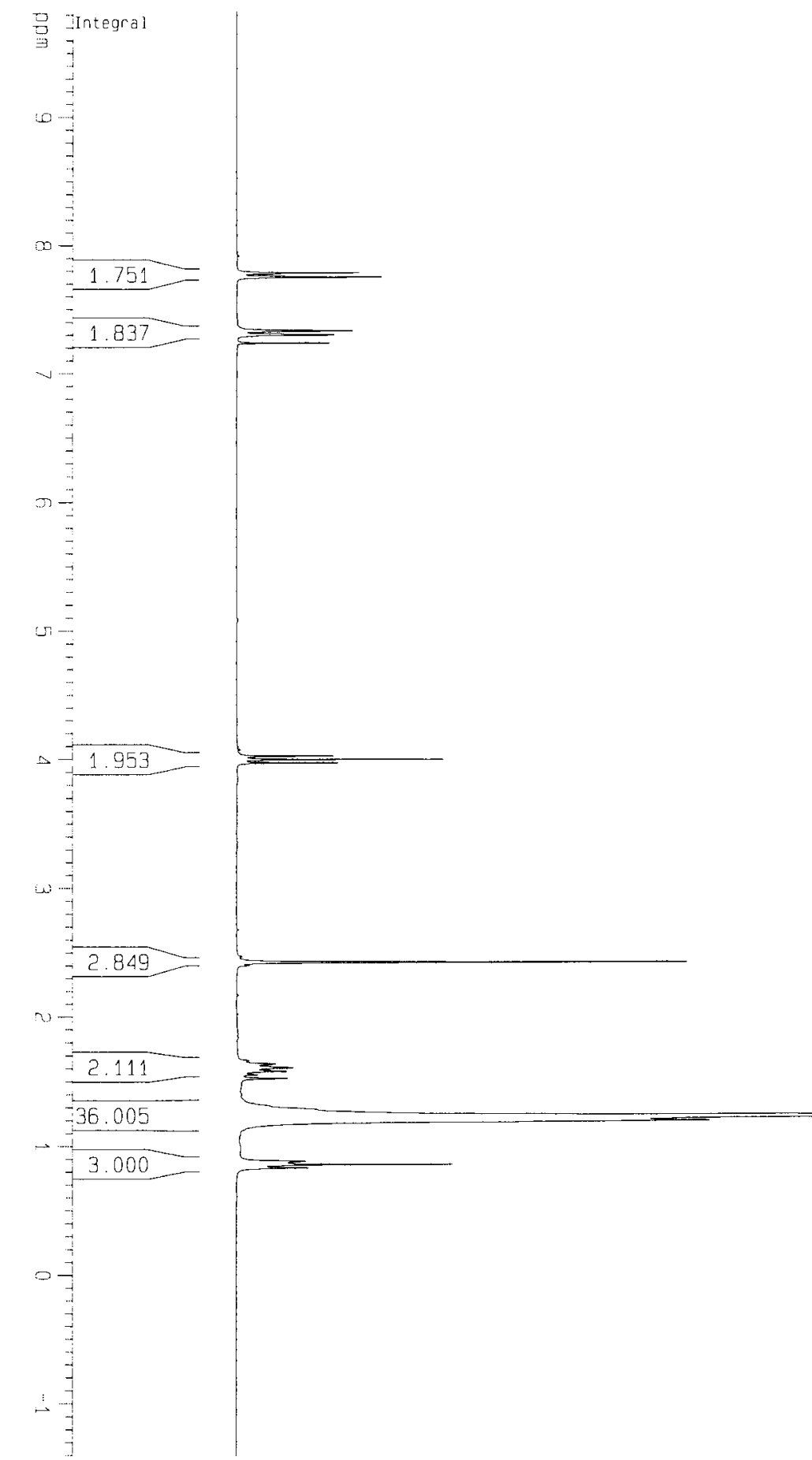



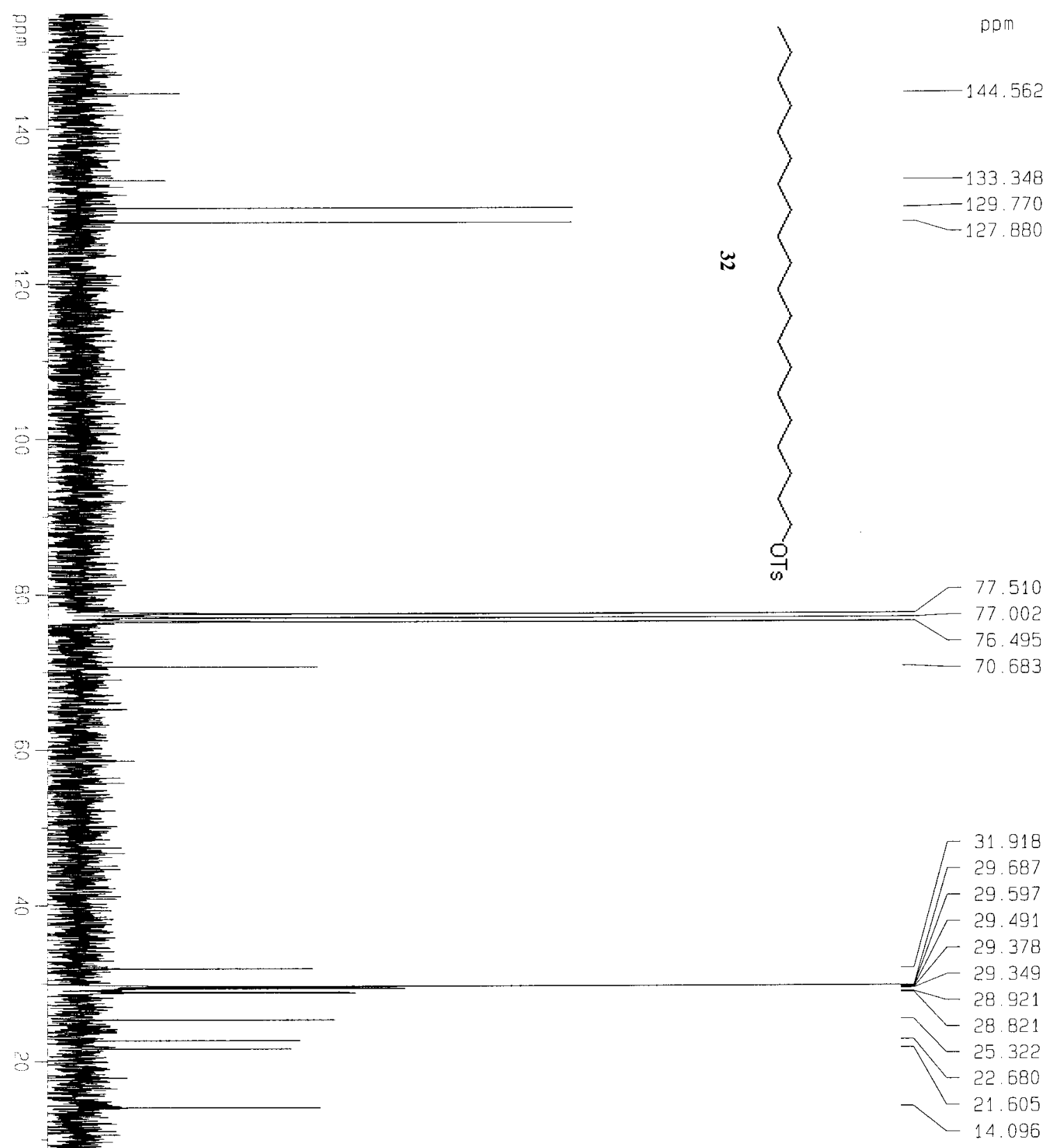

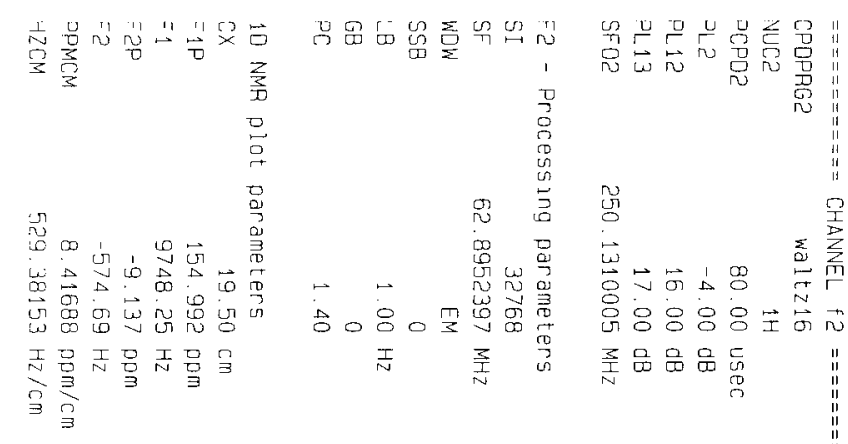

$$
\text { 政 }
$$

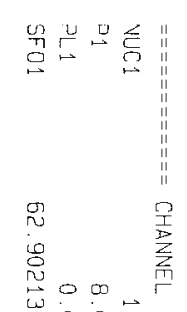

过 $8 \vec{\circ} \omega$

㞼吕㫐

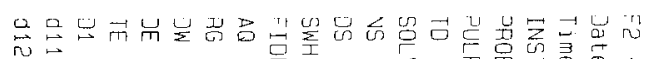

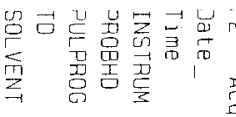

o on

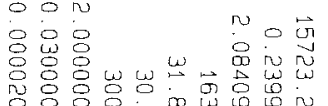

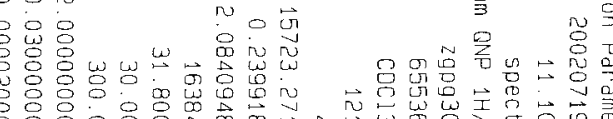

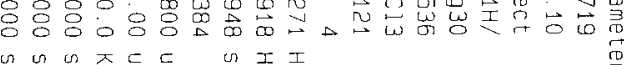

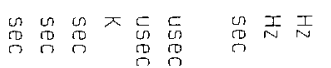

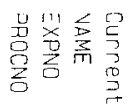
$\frac{9}{\square}$

ก.

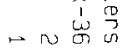

